\title{
ACUMULACIÓN DE RESERVAS INTERNACIONALES A TRAVÉS DE LA VENTA DE OPCIONES: EL CASO DE MÉXICO.
}

\author{
Alejandro Werner \\ Alexis Milo ${ }^{1}$ \\ Abril de 1998 \\ Documento de Investigación No. 9801 \\ Dirección General de Investigación Económica \\ Banco de México
}

${ }^{1}$ Los autores agradecen los comentarios de Agustín Carstens, Javier Duclaud, Manuel Galán, Alonso García, Juan Manuel Pérez Porrúa y Moisés Schwartz. Las opiniones contenidas en el presente documento reflejan los puntos de vista de los autores y no necesariamente coinciden con los del Banco de México. 


\title{
ACUMULACIÓN DE RESERVAS INTERNACIONALES A TRAVÉS DE LA VENTA DE OPCIONES: EL CASO DE MÉXICO.
}

\author{
Alejandro Werner \\ Alexis Milo \\ Banco de México \\ awerner@banxico.org.mx
}

Resumen

\begin{abstract}
En este trabajo se describe el esquema de acumulación de reservas internacionales mediante la venta de opciones de venta de dólares implementado por el Banco de México. Además se presenta un modelo de valuación de dicha opción y se establece la función de ejercicio óptimo. Con base en este modelo se investigan las características principales de este mecanismo. Se demuestra que la probabilidad de ejercicio de la opción no se ve afectada de manera importante por cambios en la volatilidad y la tendencia del tipo de cambio. Por lo tanto, se concluye que este mecanismo ha sido neutral a cambios en estos parámetros. Finalmente, se compara la valuación y el momento de ejercicio determinados teóricamente con las observaciones de mercado. En dicha comparación se hace evidente una subvaluación del precio de la opción y un ejercicio acelerado de la opción en relación a lo indicado por el modelo.
\end{abstract}




\section{I.- Introducción:}

Este trabajo describe un esquema de acumulación de reservas internacionales y de intervención en el mercado cambiario a través de la colocación por parte de la autoridad, de opciones de venta de dólares, implementado recientemente por el Banco de México. En el trabajo se desarrolla una metodología para valuar la opción de venta de dólares emitida por parte del Banco de México y determinar el momento óptimo de ejercicio de dicha opción. Además, esta metodología nos permite calcular la probabilidad de ejercicio de la opción y evaluar la sensibilidad del precio, el momento de ejercicio y la probabilidad de ejercicio ante cambios en los parámetros del proceso seguido por el tipo de cambio. De esta manera se evalúan las propiedades principales de este esquema.

$\mathrm{Al}$ implementarse el régimen de flotación en el país se temía que la cotización de la moneda extranjera resultara muy volátil. Sin embargo, la evolución del tipo de cambio a partir de principios de 1996 ha disipado este temor. Si bien es cierto que durante 1995 la cotización del peso frente al dólar mostró una gran inestabilidad, ésta fue resultado del ambiente generalizado de incertidumbre macroeconómica y no de las características del régimen de flotación.

La volatilidad de la cotización del peso se redujo en forma considerable durante 1996 y 1997. Según una muestra que incluye 12 divisas importantes en flotación frente al dólar, la tasa de cambio del peso mexicano fue la segunda menos volátil en 1996 y la menos volátil en el primer semestre de 1997 (ver Cuadro 1).

Este fenómeno ha sido resultado de un entorno internacional favorable, una política fiscal atinada, la continuación del proceso de desregulación y privatización, y una política monetaria conducente al abatimiento de la inflación. Otro factor importante que ha contribuido a la estabilidad del peso ha sido el desarrollo y profundización del mercado cambiario y de derivados del peso. Como ejemplo de esto se ha observado que el volumen promedio diario de operación en el mercado alcanza los 9,000 millones de dólares, lo cual representa un aumento de $270 \%$ respecto a mediados de 1995. A su vez, el volumen de contratos abiertos en el mercado de futuros del Peso mexicano en Chicago aumentó en 262\% entre 1995 y 1997.

No obstante, el renovado nerviosismo ocasionado por la crisis de los países asiáticos propició que la tasa de cambio del peso mexicano pasara a ser la segunda más volátil de la muestra durante el segundo semestre de 1997. Sin embargo, a diferencia de lo observado en 1995, la volatilidad del peso en el segundo semestre de 1997 es del mismo orden de magnitud que la observada en los otros países de la muestra. 


\section{Cuadro 1}

VOLATILIDAD DE LOS TIPOS DE CAMBIO DE LAS MONEDAS NACIONALES DE ALGUNOS PAISES FRENTE AL DÓLAR

\begin{tabular}{|c|c|c|c|c|c|c|c|}
\hline & \multicolumn{7}{|c|}{ Volatilidad Anualizada* (En porciento) } \\
\hline & $1995-I$ & 1995-II & $1996-I$ & 1996-II & $1997-I$ & 1997-|| & $1998-I^{\star \star}$ \\
\hline México & $48.56 \%$ & $17.60 \%$ & $5.99 \%$ & $5.31 \%$ & $4.92 \%$ & $10.69 \%$ & $7.88 \%$ \\
\hline Nueva Zelandia & $6.67 \%$ & $5.35 \%$ & $5.56 \%$ & $6.01 \%$ & $5.88 \%$ & $8.61 \%$ & $13.91 \%$ \\
\hline Australia & $8.86 \%$ & $7.87 \%$ & $6.10 \%$ & $6.70 \%$ & $7.65 \%$ & $10.29 \%$ & $13.19 \%$ \\
\hline Finlandia & $12.85 \%$ & $9.13 \%$ & $7.43 \%$ & $7.28 \%$ & $10.04 \%$ & $9.51 \%$ & $9.43 \%$ \\
\hline Suecia & $12.70 \%$ & $9.91 \%$ & $8.02 \%$ & $7.28 \%$ & $10.26 \%$ & $10.58 \%$ & $10.09 \%$ \\
\hline Canadá & $5.13 \%$ & $5.92 \%$ & $3.64 \%$ & $3.33 \%$ & $5.46 \%$ & $4.24 \%$ & $4.73 \%$ \\
\hline Italia & $13.97 \%$ & $7.22 \%$ & $5.97 \%$ & $5.73 \%$ & $8.95 \%$ & $9.15 \%$ & $8.85 \%$ \\
\hline Sudáfrica & $5.64 \%$ & $2.34 \%$ & $13.86 \%$ & $7.20 \%$ & $5.25 \%$ & $4.34 \%$ & $4.66 \%$ \\
\hline Reino Unido & $10.45 \%$ & $7.41 \%$ & $5.60 \%$ & $6.30 \%$ & $8.13 \%$ & $8.03 \%$ & $8.22 \%$ \\
\hline Suiza & $16.67 \%$ & $12.13 \%$ & $7.77 \%$ & $8.84 \%$ & $11.70 \%$ & $9.81 \%$ & $9.75 \%$ \\
\hline Japón & $14.12 \%$ & $14.04 \%$ & $8.14 \%$ & $7.09 \%$ & $12.56 \%$ & $12.29 \%$ & $11.95 \%$ \\
\hline Alemania & $13.87 \%$ & $10.89 \%$ & $6.28 \%$ & $6.87 \%$ & $9.79 \%$ & $9.76 \%$ & $9.36 \%$ \\
\hline
\end{tabular}

* La volatilidad anualizada se define como la desviación estándar anualizada de las

variaciones diarias del tipo de cambio.

${ }^{* *}$ Hasta el 2 de abril de 1998

Debido a la evolución favorable del mercado cambiario en 1996 y a la gran caída que habían sufrido las reservas internacionales del Banco de México como consecuencia de la crisis de diciembre de 1994, la Comisión de Cambios ${ }^{2}$ consideró, en agosto de 1996, que por motivos prudenciales sería conveniente aumentar el nivel de reservas del instituto emisor.

Un monto mayor de activos internacionales contribuiría a que el país obtuviese mejores condiciones en cuanto al plazo y al costo del financiamiento externo. Además, la Comisión de Cambios consideró que esta acumulación se podría lograr sin influir el funcionamiento del mercado cambiario. Se advirtió la importancia de que esta acumulación se logre mediante un esquema que favorezca las compras del Banco de México cuando el mercado esté ofrecido y las inhiba cuando esté demandado. Sobre todo, se estimó de suma importancia que mediante estas compras no se envíen señales sobre el nivel del tipo de cambio, preservándose así una de las características principales del régimen de libre flotación.

En base a estas consideraciones se estableció un programa de adquisición de divisas a través de la venta de opciones de venta de dólares por parte del propio Banco de México ${ }^{3}$. En primer lugar, se decidió que las subastas mensuales de opciones serían de 130 millones de dólares, monto que se aumentó inmediatamente a

\footnotetext{
${ }^{2}$ La Comisión de Cambios está conformada por el gobernador y dos subgobernadores del Banco de México y el secretario y dos subsecretarios de la Secretaría de Hacienda y Crédito Público. Esta comisión es la responsable de todas las decisiones relacionadas con la política cambiaria.

${ }^{3}$ En la sección 2 se hace una descripción detallada del funcionamiento de estos instrumentos.
} 
200 millones de dólares para septiembre de 1996 y a 300 millones de dólares en diciembre de ese año. En febrero de 1997, la Comisión de Cambios resolvió que a partir de ese mes, en caso de ejercerse antes del día 16 el 80\% o más de las opciones correspondientes a ese mes, se convocaría inmediatamente a una subasta adicional de opciones por otros 300 millones de dólares. Estas últimas tendrían vigencia sólo por el resto del mes respectivo. A finales de julio de 1997, la Comisión de Cambios decidió incrementar a 500 millones de dólares la subasta de opciones susceptibles de ejercerse en agosto, realizándose una subasta por igual cantidad si, durante la primera quincena, se hubieran ejercido $80 \%$ o más de las opciones subastadas el 31 de julio. Este último monto continuó vigente durante septiembre. En virtud de la rápida acumulación de activos internacionales que se venía observando, la Comisión de Cambios acordó reducir el monto de opciones de venta de dólares al Banco de México que podrían ejercerse durante el mes de octubre a 400 m.d.d.. Finalmente, el 30 de octubre estas opciones se redujeron nuevamente a 250 m.d.d., lo cual representaba un monto potencial de compra de dólares por parte del Banco de México de 500 m.d.d. al mes. El monto señalado se mantiene vigente hasta la fecha.

El mecanismo de opciones ha cumplido el objetivo para el cual fue creado. Durante los 20 meses de operación el Banco de México ha adquirido, a través de este mecanismo, 5,644 millones de dólares. El resumen de estas compras se presenta en el Cuadro 2. 


\begin{tabular}{|c|c|c|c|}
\hline \multicolumn{4}{|c|}{$\begin{array}{c}\text { Cuadro } 2 \\
\text { Resultado de las Subastas de Opciones }\end{array}$} \\
\hline $\begin{array}{c}\text { Fecha } \\
\text { de la } \\
\text { Subasta }\end{array}$ & $\begin{array}{c}\text { Monto } \\
\text { Subastado } \\
\text { (MDD) }\end{array}$ & $\begin{array}{c}\text { Monto } \\
\text { Ejercido } \\
\text { (MDD) }\end{array}$ & $\begin{array}{c}\text { Proporción } \\
\text { Ejercida }\end{array}$ \\
\hline 7-Ago-96 & 130 & 130 & $100.0 \%$ \\
\hline 30-Ago-96 & 200 & 200 & $100.0 \%$ \\
\hline 30-Sep-96 & 200 & 179 & $89.5 \%$ \\
\hline 31-Oct-96 & 200 & 200 & $100.0 \%$ \\
\hline 29-Nov-96 & 200 & 200 & $100.0 \%$ \\
\hline 30-Dic-96 & 300 & 300 & $100.0 \%$ \\
\hline 31-Ene-97 & 300 & 300 & $100.0 \%$ \\
\hline 21-Feb-97 & 300 & 148 & $49.3 \%$ \\
\hline 28-Feb-97 & 300 & 120 & $40.0 \%$ \\
\hline 31-Mar-97 & 300 & 300 & $100.0 \%$ \\
\hline 15-Abr-97 & 300 & 263 & $87.7 \%$ \\
\hline $30-A b r-97$ & 300 & 300 & $100.0 \%$ \\
\hline 30-May-97 & 300 & 20 & $6.7 \%$ \\
\hline 30-Jun-97 & 300 & 300 & $100.0 \%$ \\
\hline 9-Jul-97 & 300 & 300 & $100.0 \%$ \\
\hline 31-Jul-97 & 500 & 460 & $92.0 \%$ \\
\hline 29-Ago-97 & 500 & 500 & $100.0 \%$ \\
\hline 30-Sep-97 & 400 & 375 & $93.8 \%$ \\
\hline 31-Oct-97 & 250 & 250 & $100.0 \%$ \\
\hline 28-Nov-97 & 250 & 250 & $100.0 \%$ \\
\hline 15-Dic-97 & 250 & 250 & $100.0 \%$ \\
\hline 30-Dic-97 & 250 & 49 & $19.6 \%$ \\
\hline 30-Ene-98 & 250 & 0 & $0.0 \%$ \\
\hline 27-Feb-98 & 250 & 250 & $100.0 \%$ \\
\hline Total & 6830 & 5644 & $82.6 \%$ \\
\hline
\end{tabular}

El trabajo se estructura de la siguiente manera; en la sección II se hace una descripción detallada de la opción de compra de dólares y su funcionamiento hasta la fecha. En la sección III presentamos el modelo de valuación, y se calculan la función de ejercicio óptimo y la probabilidad de ejercicio. En esta sección también se realizan varios ejercicios que estiman la sensibilidad de los resultados a cambios en los parámetros. En la sección IV se realiza una evaluación empírica de nuestros resultados y se comparan las estimaciones con los precios y el ejercicio observado durante la vigencia del programa. Finalmente, la sección V concluye.

\section{II.- El programa de acumulación de activos internacionales a través de la venta de opciones.}

Como se mencionó en la introducción, las consideraciones principales en el diseño de un mecanismo de acumulación de reservas eran el no influir sobre el 
comportamiento del mercado cambiario, que las compras se lleven a cabo cuando el mercado esté ofrecido y por último, el evitar que mediante estas compras se envíen señales sobre niveles del tipo de cambio que pudieran ser interpretados como "niveles objetivo" de la autoridad. Con base en estas consideraciones el 1 de agosto de 1996, el Banco de México adoptó un esquema de acumulación de reservas internacionales cuyos elementos principales se resumen a continuación.

a) El Banco de México subastará el último día hábil de cada mes entre las instituciones de crédito derechos de venta de dólares al propio Banco. Estos derechos se podrán ejercer total o parcialmente durante el mes inmediato siguiente al de la subasta respectiva.

b) Los tenedores de los derechos podrán vender dólares al Banco de México al tipo de cambio interbancario de referencia determinado el día hábil inmediato anterior, cuando dicho tipo de cambio no sea mayor a su promedio de los veinte días hábiles inmediatos anteriores al día del ejercicio de los derechos respectivos.

c) Las compras de divisas producto del esquema de opciones serán esterilizadas en su totalidad.

En el inciso (b) se indica que el tipo de cambio de ejercicio de la opción cambia día con día y es igual al tipo de cambio de referencia ${ }^{4}$ del día anterior. Esto indica que la ganancia obtenida en el ejercicio de esta opción será igual a la apreciación diaria del tipo de cambio. En segundo lugar, se especifica que la opción sólo se podrá ejercer si el tipo de cambio de ejercicio es mayor que el promedio móvil de este tipo de cambio para los 20 días hábiles inmediatos anteriores. Esta restricción evita que de darse una depreciación abrupta del tipo de cambio y una apreciación subsecuente moderada, el Banco de México acumule reservas en un momento en el cual la liquidez en el mercado cambiario disminuye considerablemente debido al aumento en la incertidumbre. De presentarse esta situación se podría estar influyendo de manera importante en la determinación del tipo de cambio.

La estructura propuesta cumple con los requerimientos de no señalar ningún nivel específico para el tipo de cambio y de incentivar las compras del Banco de México cuando el mercado está ofrecido y de inhibirlas cuando se encuentra demandado. El primer requerimiento se cumple debido a que son los dueños de la opción y no el Banco de México, los que deciden cuando ejercerla. Por lo tanto, el criterio usado para determinar el ejercicio responde solamente al propósito de maximizar las ganancias de los dueños de la opción y no a algún criterio de intervención en el mercado cambiario determinado por el Banco Central. Además, la

\footnotetext{
${ }^{4}$ También llamado tipo de cambio "fix".
} 
aplicación de este criterio se basa en las expectativas del mercado sobre el curso futuro del tipo de cambio y su volatilidad y no en la expectativa que sostiene la autoridad monetaria con respecto a estos parámetros. El segundo requerimiento se cumple ya que los tenedores de la opción la ejercerán en los períodos en los que se observe una tendencia hacia la apreciación del tipo de cambio, o sea, cuando el mercado esté ofrecido.

En la Gráfica 1 se muestra la evolución del tipo de cambio "fix", su promedio móvil de los 20 días previos y el monto de opciones ejercidas, para el período en el cual ha estado en vigencia el programa de acumulación de reservas a través de la venta de opciones. Se puede ver que de los 408 días hábiles observados, en 186 ocasiones la restricción ha operado. 


\section{Gráfica 1}

Tipo de Cambio Interbancario de Referencia (Fix) y su Promedio Móvil ${ }^{\star}$

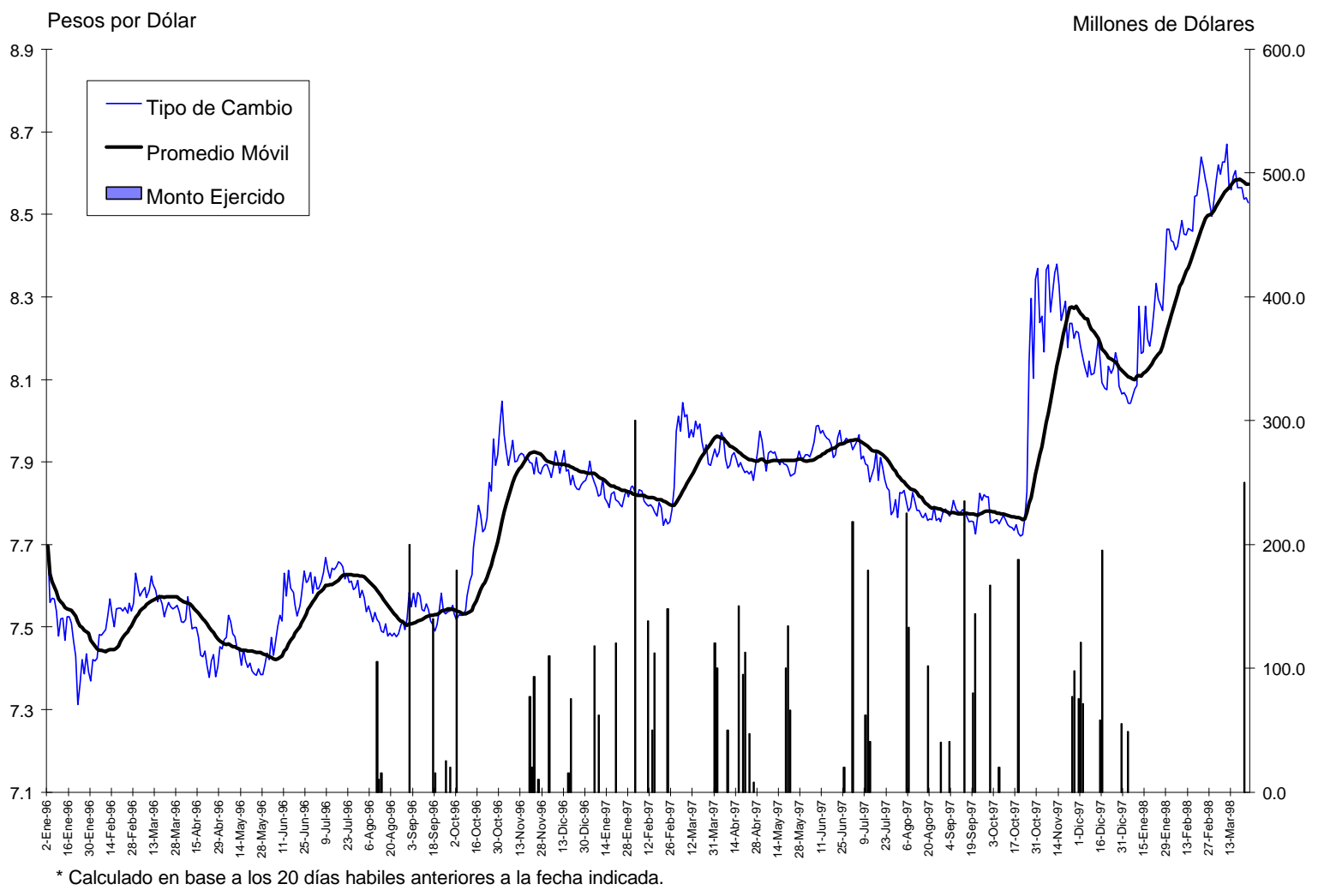

Por otro lado, en el Cuadro 3, se presenta el precio de la opción en cada subasta, las fechas y montos en los que las opciones fueron ejercidas y la utilidad obtenida por los dueños de la opción. En primer lugar vemos que la prima pagada ha fluctuado entre 33.9 y 4.6 pesos por cada mil dólares. Por otro lado, la ganancia promedio que obtuvieron los tenedores de la opción ha fluctuado entre $0.51 \%$ y $0 \%$ y la ganancia máxima ha sido de $0.80 \%$, mientras que la mínima ha sido de $0 \%$. Observando las fechas y las apreciaciones de ejercicio no se puede detectar un patrón claro que esté guiando las decisiones de ejercicio de los dueños de la opción. 


\section{Cuadro 3}

\begin{tabular}{|c|c|c|c|c|c|c|c|c|c|c|}
\hline \multirow{2}{*}{$\begin{array}{c}\text { Fecha } \\
\text { de la } \\
\text { Subasta }\end{array}$} & \multirow{2}{*}{$\begin{array}{l}\text { Vigencia } \\
\text { de la } \\
\text { Opción }\end{array}$} & \multirow{2}{*}{$\begin{array}{c}\text { Monto } \\
\text { Subastado } \\
\text { (MDD) }\end{array}$} & \multirow{2}{*}{$\begin{array}{c}\text { Prima } \\
\text { Ponderada } \\
\text { (\$ x Mil DIs) }\end{array}$} & \multirow{2}{*}{$\begin{array}{c}\text { Fecha } \\
\text { de } \\
\text { Ejercicio }\end{array}$} & \multirow{2}{*}{$\begin{array}{c}\text { Monto } \\
\text { Ejercido } \\
\text { (MDD) }\end{array}$} & \multirow{2}{*}{$\begin{array}{l}\text { Apreciación } \\
\text { del T. De C. } \\
\text { (Ganancia) }\end{array}$} & \multicolumn{4}{|c|}{ Ganancias por Subasta } \\
\hline & & & & & & & Máxima & Mínima & $\begin{array}{l}\text { Promedio } \\
\text { Ejercidas }\end{array}$ & $\begin{array}{c}\text { Promedio } \\
\text { Total }\end{array}$ \\
\hline 7-Ago-96 & Agcion & $\frac{(\text { IIVD) }}{130}$ & $\begin{array}{c}c(8 \times \text { IVIII UIS } \\
(11.68 \\
(0.16 \%)\end{array}$ & $\begin{array}{c}\text { 딜clcio } \\
\text { 9-Ago-96 } \\
\text { 12-Ago-96 } \\
\text { 13-Ago-96 } \\
\text { Subtotal }\end{array}$ & $\begin{array}{c}\text { (IVIDD) } \\
105 \\
10 \\
15 \\
130\end{array}$ & $\begin{array}{c}\text { (uanancla) } \\
0.23 \% \\
0.09 \% \\
0.28 \%\end{array}$ & $0.28 \%$ & 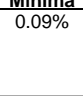 & $0.23 \%$ & $0.23 \%$ \\
\hline 30-Ago-96 & Sep-96 & 200 & $\begin{array}{c}4.65 \\
(0.06 \%)\end{array}$ & $\begin{array}{l}\text { 17-Sep-96 } \\
\text { 18-Sep-96 } \\
25-\text { Sep-96 } \\
\text { 27-Sep-96 } \\
\quad \text { Subtotal }\end{array}$ & $\begin{array}{c}140 \\
15 \\
25 \\
20 \\
200\end{array}$ & $\begin{array}{l}0.11 \% \\
0.20 \% \\
0.06 \% \\
0.00 \%\end{array}$ & $0.20 \%$ & $0.06 \%$ & $0.10 \%$ & $0.10 \%$ \\
\hline 30-Sep-96 & Oct-96 & 200 & $\begin{array}{c}10.18 \\
(0.13 \%) \\
\end{array}$ & $\begin{array}{l}2-\text { Oct-96 } \\
\text { Subtotal }\end{array}$ & $\begin{array}{l}179 \\
179 \\
\end{array}$ & $0.18 \%$ & $0.18 \%$ & $0.18 \%$ & $0.18 \%$ & $0.16 \%$ \\
\hline 31-Oct-96 & Nov-96 & 200 & $\begin{array}{l}10.32 \\
(0.13 \%)\end{array}$ & $\begin{array}{l}19-N o v-96 \\
21-N o v-96 \\
22-N o v-96 \\
26-N o v-96 \\
\quad \text { Subtotal }\end{array}$ & $\begin{array}{l}77 \\
20 \\
93 \\
10 \\
200 \\
\end{array}$ & $\begin{array}{l}0.14 \% \\
0.02 \% \\
0.33 \% \\
0.44 \%\end{array}$ & $0.44 \%$ & $0.02 \%$ & $0.23 \%$ & $0.23 \%$ \\
\hline 29-Nov-96 & Dic-96 & 200 & $\begin{array}{l}14.43 \\
(0.18 \%)\end{array}$ & $\begin{array}{c}\text { 3-Dic-96 } \\
\text { 17-Dic-96 } \\
\text { 18-Dic-96 } \\
\text { Subtotal }\end{array}$ & $\begin{array}{c}110 \\
15 \\
75 \\
\mathbf{2 0 0} \\
\end{array}$ & $\begin{array}{l}0.18 \% \\
0.00 \% \\
0.47 \%\end{array}$ & $0.47 \%$ & $0.00 \%$ & $0.27 \%$ & $0.27 \%$ \\
\hline 30-Dic-96 & Ene-97 & 300 & $\begin{array}{c}13.75 \\
(0.18 \%)\end{array}$ & $\begin{array}{l}6-\text { Ene-97 } \\
8 \text {-Ene-97 } \\
20-\text { Ene-97 } \\
\quad \text { Subtotal }\end{array}$ & $\begin{array}{c}118 \\
62 \\
120 \\
300 \\
\end{array}$ & $\begin{array}{l}0.20 \% \\
0.26 \% \\
0.25 \%\end{array}$ & $0.26 \%$ & $0.20 \%$ & $0.23 \%$ & $0.23 \%$ \\
\hline 31-Ene-96 & Feb-97 & 300 & $\begin{array}{c}16.14 \\
(0.21 \%)\end{array}$ & $\begin{array}{l}11-\text { Feb-97 } \\
13-\text { Feb-97 } \\
\text { 14-Feb-97 } \\
\text { Subtotal }\end{array}$ & $\begin{array}{c}138 \\
50 \\
112 \\
300 \\
\end{array}$ & $\begin{array}{l}0.08 \% \\
0.09 \% \\
0.15 \%\end{array}$ & $0.15 \%$ & $0.08 \%$ & $0.11 \%$ & $0.11 \%$ \\
\hline 21-Feb-97 & $\begin{array}{c}\text { Feb-97 } \\
\text { (segunda) }\end{array}$ & 300 & $\begin{array}{c}15.08 \\
(0.19 \%) \\
\end{array}$ & $\begin{array}{c}24-F e b-97 \\
\text { Subtotal }\end{array}$ & $\begin{array}{l}148 \\
148 \\
\end{array}$ & $0.15 \%$ & $0.15 \%$ & $0.15 \%$ & $0.15 \%$ & $0.07 \%$ \\
\hline 28-Feb-97 & Mar-97 & 300 & $\begin{array}{c}10.4 \\
(0.13 \%) \\
\end{array}$ & $\begin{array}{c}\text { 31-Mar-97 } \\
\text { Subtotal }\end{array}$ & $\begin{array}{l}120 \\
120 \\
\end{array}$ & $0.00 \%$ & $0.00 \%$ & $0.00 \%$ & $0.00 \%$ & $0.00 \%$ \\
\hline 31-Mar-97 & Abr-97 & 300 & $\begin{array}{l}21.86 \\
(0.28 \%)\end{array}$ & $\begin{array}{l}1-A b r-97 \\
8-A b r-97 \\
15-A b r-97 \\
\quad \text { Subtotal }\end{array}$ & $\begin{array}{c}100 \\
50 \\
150 \\
300 \\
\end{array}$ & $\begin{array}{l}0.25 \% \\
0.27 \% \\
0.24 \%\end{array}$ & $0.27 \%$ & $0.24 \%$ & $0.24 \%$ & $0.24 \%$ \\
\hline 15-Abr-97 & $\begin{array}{c}\text { Abr-97 } \\
\text { (segunda) }\end{array}$ & 300 & $\begin{array}{c}13.86 \\
(0.18 \%)\end{array}$ & \begin{tabular}{l} 
17-Abr-97 \\
$18-A b r-97$ \\
$22-A b r-97$ \\
$24-A b r-97$ \\
\multicolumn{1}{c}{ Subtotal }
\end{tabular} & $\begin{array}{c}95 \\
95 \\
113 \\
47 \\
8 \\
263 \\
\end{array}$ & $\begin{array}{l}0.16 \% \\
0.14 \% \\
0.09 \% \\
0.30 \%\end{array}$ & $0.30 \%$ & $0.09 \%$ & $0.14 \%$ & $0.13 \%$ \\
\hline 30-Abr-97 & May-97 & 300 & $\begin{array}{c}14.38 \\
(0.18 \%)\end{array}$ & $\begin{array}{l}19-\text { May-97 } \\
20-\text { May-97 } \\
21 \text {-May-97 } \\
\text { Subtotal }\end{array}$ & $\begin{array}{c}100 \\
134 \\
66 \\
300\end{array}$ & $\begin{array}{l}0.01 \% \\
0.07 \% \\
0.28 \%\end{array}$ & $0.28 \%$ & $0.01 \%$ & $0.10 \%$ & $0.10 \%$ \\
\hline 30-May-97 & Jun-97 & 300 & $\begin{array}{c}11.74 \\
(0.15 \%)\end{array}$ & $\begin{array}{l}25-J u n-97 \\
\text { Subtotal }\end{array}$ & $\begin{array}{l}20 \\
20 \\
\end{array}$ & $0.00 \%$ & $0.00 \%$ & $0.00 \%$ & $0.00 \%$ & $0.00 \%$ \\
\hline 30-Jun-97 & Jul-97 & 300 & $\begin{array}{l}20.61 \\
(0.26 \%)\end{array}$ & $\begin{array}{l}1-J u l-97 \\
\text { 9-Jul-97 } \\
11-J u l-97 \\
\quad \text { Subtotal }\end{array}$ & $\begin{array}{c}218 \\
62 \\
20 \\
300 \\
\end{array}$ & $\begin{array}{l}0.26 \% \\
0.25 \% \\
0.49 \%\end{array}$ & $0.49 \%$ & $0.25 \%$ & $0.27 \%$ & $0.27 \%$ \\
\hline 9-Jul-97 & $\begin{array}{c}\text { Jul-97 } \\
\text { (segunda) }\end{array}$ & 300 & $\begin{array}{c}19.57 \\
(0.25 \%)\end{array}$ & $\begin{array}{c}10-\text { Jul-97 } \\
11 \text {-Jul-97 } \\
\text { Subtotal }\end{array}$ & $\begin{array}{c}300 \\
279 \\
21 \\
300 \\
\end{array}$ & $\begin{array}{l}0.05 \% \\
0.49 \%\end{array}$ & $0.49 \%$ & $0.05 \%$ & $0.08 \%$ & $0.08 \%$ \\
\hline 31-Jul-97 & Ago-97 & 500 & $\begin{array}{c}23.36 \\
(0.30 \%)\end{array}$ & $\begin{array}{l}\text { 5-Ago-97 } \\
\text { 6-Ago-97 } \\
\text { 19-Ago-97 } \\
\text { 27-Ago-97 } \\
\quad \text { Subtotal }\end{array}$ & $\begin{array}{c}225 \\
133 \\
102 \\
40 \\
\mathbf{5 0 0} \\
\end{array}$ & $\begin{array}{l}0.33 \% \\
0.31 \% \\
0.23 \% \\
0.11 \%\end{array}$ & $0.33 \%$ & $0.11 \%$ & $0.29 \%$ & $0.29 \%$ \\
\hline 29-Ago-97 & Sep-97 & 500 & $\begin{array}{l}12.27 \\
(0.16 \%)\end{array}$ & $\begin{array}{l}\text { 3-Sep-97 } \\
17-\text { Sep-97 } \\
19-\text { Sep-97 } \\
\text { 22-Sep-97 } \\
\quad \text { Subtotal }\end{array}$ & $\begin{array}{c}41 \\
235 \\
80 \\
144 \\
500\end{array}$ & $\begin{array}{l}0.11 \% \\
0.17 \% \\
0.00 \% \\
0.40 \%\end{array}$ & $0.40 \%$ & $0.00 \%$ & $0.20 \%$ & $0.20 \%$ \\
\hline 30-Sep-97 & Oct-97 & 400 & $\begin{array}{c}16.10 \\
(0.21 \%)\end{array}$ & $\begin{array}{l}1-\text { Oct-97 } \\
7-\text { Oct-97 } \\
20-\text { Oct-97 } \\
\text { Subtotal } \\
\end{array}$ & $\begin{array}{c}167 \\
20 \\
188 \\
375 \\
\end{array}$ & $\begin{array}{l}0.80 \% \\
0.00 \% \\
0.37 \%\end{array}$ & $0.80 \%$ & $0.00 \%$ & $0.54 \%$ & $0.51 \%$ \\
\hline 31-Oct-97 & Nov-97 & 250 & $\begin{array}{c}13.05 \\
(0.16 \%)\end{array}$ & $\begin{array}{l}\text { 25-Nov-97 } \\
26-N o v-97 \\
28-N o v-97 \\
\quad \text { Subtotal }\end{array}$ & $\begin{array}{c}77 \\
98 \\
75 \\
250\end{array}$ & $\begin{array}{l}0.00 \% \\
0.43 \% \\
0.04 \%\end{array}$ & $0.43 \%$ & $0.00 \%$ & $0.18 \%$ & $0.18 \%$ \\
\hline 28-Nov-97 & Dic-97 & 250 & $\begin{array}{l}30.50 \\
(0.37 \%)\end{array}$ & $\begin{array}{l}1-\text { Dic-97 } \\
2 \text {-Dic-97 } \\
\text { 15-Dic-97 } \\
\quad \text { Subtotal }\end{array}$ & $\begin{array}{c}121 \\
71 \\
58 \\
250 \\
\end{array}$ & $\begin{array}{l}0.40 \% \\
0.33 \% \\
0.76 \%\end{array}$ & $0.76 \%$ & $0.33 \%$ & $0.46 \%$ & $0.46 \%$ \\
\hline 15-Dic-97 & Dic-97 & 250 & $\begin{array}{c}33.81 \\
(0.42 \%)\end{array}$ & $\begin{array}{l}16 \text {-Dic-97 } \\
\text { 30-Dic-97 } \\
\quad \text { Subbtotal }\end{array}$ & $\begin{array}{c}195 \\
55 \\
250\end{array}$ & $\begin{array}{l}0.57 \% \\
0.24 \%\end{array}$ & $0.57 \%$ & $0.24 \%$ & $0.49 \%$ & $0.49 \%$ \\
\hline 30-Dic-97 & Ene-98 & 250 & $\begin{array}{c}33.54 \\
(0.42 \%)\end{array}$ & $\begin{array}{l}\text { 5-Ene-98 } \\
\text { Subtotal }\end{array}$ & $\begin{array}{l}49 \\
49\end{array}$ & $0.20 \%$ & $0.20 \%$ & $0.20 \%$ & $0.20 \%$ & $0.04 \%$ \\
\hline 30-Ene-98 & Feb-98 & 250 & $\begin{array}{l}15.85 \\
(0.19 \%)\end{array}$ & $\begin{array}{c}\text { No Ejercidas } \\
\text { Subtotal }\end{array}$ & $\begin{array}{l}3 \\
0 \\
0\end{array}$ & $0.00 \%$ & $0.00 \%$ & $0.00 \%$ & $0.00 \%$ & $0.00 \%$ \\
\hline 27-Feb-98 & Mar-98 & 250 & $\begin{array}{l}30.25 \\
(0.35 \%) \\
\end{array}$ & $\begin{array}{c}\text { 23-Mar-98 } \\
\text { Subtotal }\end{array}$ & $\begin{array}{l}250 \\
250 \\
\end{array}$ & $0.33 \%$ & $0.33 \%$ & $0.33 \%$ & $0.33 \%$ & $0.33 \%$ \\
\hline
\end{tabular}




\section{III.- Modelo de Valuación:}

En esta sección se presenta la metodología de valuación del precio de la opción y de determinación del momento óptimo de ejercicio. Esta metodología es un aplicación sencilla del método de programación dinámica ${ }^{5}$.

Para valuar esta opción suponemos que el logaritmo del tipo de cambio sigue una caminata al azar con tendencia. Varios estudios ${ }^{6}$ han demostrado que ésta es la mejor caracterización del proceso seguido por las monedas que se encuentran en flotación, incluyendo al peso.

$$
\text { (1) } S_{t}=\gamma+S_{t-1}+\varepsilon_{t}
$$

Donde $S_{t}$ es el logaritmo del tipo de cambio en el período $t, \gamma$ es su tendencia y $\varepsilon_{t}$ es un error con media cero y varianza $\sigma^{2}$. Si definimos el promedio móvil del nivel del tipo de cambio como:

( 2) $\quad M O V_{t}=\frac{1}{20} \sum_{i=0}^{19} E_{t-i} \quad$ y $\quad M_{t}=\operatorname{Ln}\left(M O V_{t}\right)$

En donde $E_{t}$ es el nivel del tipo de cambio, $M O V_{t}$ es el promedio móvil y $M_{t}$ es el logaritmo de este promedio móvil. Por lo tanto, tenemos que siempre se cumple la siguiente igualdad:

(3) $\frac{M O V_{t}}{E_{t}}=\frac{M O V_{t-1}\left(1+\Delta M_{t}\right)}{E_{t-1}\left(1+\Delta S_{t}\right)}$.

Tomando logaritmo de ambos lados se obtiene:

$$
\operatorname{Ln}\left(M O V_{t}\right)-\operatorname{Ln}\left(E_{t}\right)=M_{t}-S_{t}=\operatorname{Ln}\left(M O V_{t-1}\right)-\operatorname{Ln}\left(E_{t-1}\right)+\Delta M_{t}-\Delta S_{t}
$$

Si suponemos que $\Delta M_{t}=a\left(M_{t-1}-S_{t-1}\right)+b \Delta S_{t}{ }^{7}$, la ecuación (4) se puede aproximar de la siguiente manera:

\footnotetext{
${ }^{5}$ Para una descripción detallada de esta metodología veasé Sargent (1989).

${ }^{6}$ Veasé Meese y Rogoff (1983) y Werner (1997).

${ }^{7}$ Esta ecuación se estima en el apéndice 1, donde también se demuestra que los coeficientes a y b han sido extremadamente estables durante el período de agosto de 1996 a noviembre de 1997.
} 


$$
M_{t}-S_{t} \approx(1-a)\left(M_{t-1}-S_{t-1}\right)-(1-b) \Delta S_{t}
$$

Por lo tanto, suponemos que la diferencia entre el logaritmo del promedio móvil y del nivel del tipo de cambio se determina de acuerdo a la siguiente ecuación ${ }^{8}$ :

$$
\text { (6) } \quad M_{t}-S_{t}=\alpha\left(M_{t-1}-S_{t-1}\right)-\beta \Delta S_{t}
$$

En cada período $t$ la opción puede ejercerse siempre que la diferencia entre el promedio de los 20 días anteriores (hasta $t$-1) y el nivel del tipo de cambio en el período $t-1$ sea mayor que cero, $M_{t-1}-S_{t-1}>0$, y cuando se observe una apreciación del tipo de cambio el día del ejercicio $\Delta S_{t}<0$. Por lo tanto, el valor de la opción en el período $\mathrm{t}$, una vez que se conoce la realización de $\Delta S_{t}$, estará determinado por:

( 7$) \quad V\left(M_{t-1}-S_{t-1,}, \Delta S_{t}, t\right)=\left\{\begin{array}{l}\operatorname{Max}\left[-\Delta S_{t}, E V\left(M_{t}-S_{t}, t+1\right)\right] \text { Si } M_{t-1}-S_{t-1}>0 \\ E V\left(M_{t}-S_{t}, t+1\right) \quad \text { Si } M_{t-1}-S_{t-1}<0\end{array}\right.$

En donde la función $V$ representa el valor de la opción en el período $t$, dado $M_{t-1}-S_{t-1}$ y $\Delta S_{t}$. Esta expresión dice que el valor de la opción en el período $t$, cuando se sabe la realización de $\Delta S_{t}$, se calcula de la siguiente manera ${ }^{9}$ :

a) Si la restricción no opera, o sea que $M_{t-1}-S_{t-1}>0$, tendremos que el valor de la opción es el máximo de ejercer la opción hoy, en cuyo caso el dueño de la opción gana $-\Delta S_{t}$ por dólar ejercido, o esperar hasta el período siguiente. El valor de esta segunda alternativa es igual al valor esperado que tome la opción en ese período, que dado un valor de $M_{t}-S_{t}$, se calcula sobre todas las posibles realizaciones de $\Delta S_{t+1}$.

b) En caso de que la restricción opere, tendremos que el valor de la opción será igual al valor esperado de la opción en el período siguiente, ya que durante el período presente no se puede ejercer la opción. Dada la realización de $\Delta S_{t}$, se

\footnotetext{
${ }^{8}$ En donde $\alpha=1-a$ y $\beta=1-b$.

${ }^{9}$ En la descripción se omite el descuento que se lleva a cabo al comparar los valores de la opción en los días consecutivos.
} 
calcula para este valor de $M_{t}-S_{t}$ el valor esperado sobre las posibles realizaciones de $\Delta S_{t+1}$.

La decisión de ejercer o no la opción se tomará de acuerdo al siguiente criterio: siempre y cuando la restricción no opere, la opción se ejercerá cuando el beneficio de ejercerla, la apreciación del tipo de cambio, sea mayor que el valor de esperar, que es igual al valor esperado de la opción en el período siguiente.

Este problema de programación dinámica se resuelve de manera muy sencilla. Si comenzamos por el último período, dado que el valor esperado de esperarse es cero, la opción se ejercerá siempre que se pueda, esto es, siempre que $M_{19}-S_{19}>0$ y $\Delta S_{20}<0$. Con este resultado podemos calcular el valor de esperarse durante el período 19, que será igual al retorno esperado del período 20. Expresando este concepto en fórmulas tenemos que:

$$
E V\left(M_{19}-S_{19}, 20\right)=\left\{\begin{array}{l}
\int_{-\infty}^{0}-\Delta S_{t} f\left(\Delta S_{t}\right) d \Delta S_{t} \quad \text { Si } M_{19}-S_{19}>0 \\
0 \text { Si } M_{19}-S_{19}<0
\end{array}\right.
$$

Esta expresión indica que el valor de la opción cuando $M_{19}-S_{19}$ es negativo es igual a cero, ya que independientemente de la realización de $\Delta S_{20}$ la opción no se podrá ejercer. Por otro lado, cuando $M_{19}-S_{19}$ es positivo, el valor de la opción en el período 19 es igual al valor esperado de las apreciaciones en el período 20, este valor esperado se calcula como el producto de todas las posibles apreciaciones del tipo de cambio en el período 20, multiplicados por la probabilidad de que se den estas apreciaciones.

Una vez calculado este valor se puede calcular la función de ejercicio en el período 19, esta función nos dará para cada $M_{18}-S_{18}$ un nivel de $\Delta S_{19}$ que nos dice el nivel de apreciación mínima observada en el período 19 necesaria para que se ejerza la opción. Si la apreciación observada es menor que este nivel, los dueños de la opción preferirán esperarse hasta el período 20. Esto se calcularía de la siguiente manera; una vez que obtenemos $E V\left(M_{19}-S_{19}, 20\right)$, sabemos que en el período 19 ejerceremos siempre y cuando $-\Delta S_{t}>E V\left(M_{19}-S_{19}, 20\right)$

La ecuación $E V\left(M_{19}-S_{19}, 20\right)$ asigna, para cada valor de $M_{19}-S_{19}$, el valor esperado de esperarse hasta el período 20 y ejercer la opción en ese período en el 
caso que sea posible.

Por lo tanto, continuando con la recursión tenemos que dado un valor de $M_{18}-S_{18}$, tendremos que el valor esperado de no ejercer la opción y esperarse hasta el período 19 es igual a:

(9)

$$
E V\left(M_{18}-S_{18}, 19\right)= \begin{cases}\int_{-\infty}^{-E V} \Delta S_{t} f\left(\Delta S_{t}\right) d \Delta S_{t}+ & \\ \int_{-E V\left(M_{19}-S_{19}, 20\right)}^{\infty} f\left(\Delta S_{t}\right) d \Delta S_{t}\left[E V\left(M_{19}-S_{19}, 20\right)\right] & \text { Si } M_{18}-S_{18}>0 \\ E V\left(M_{19}-S_{19}, 20\right) & \text { Si } M_{18}-S_{18}<0\end{cases}
$$

Esta ecuación nos dice que el valor esperado de no ejercer la opción durante el período 18 se determina de la siguiente manera: 19.

i) Si la restricción opera, es igual al valor esperado de esperarse en el período

ii) Si la restricción no opera, es igual a la suma del valor esperado de ejercer la opción, lo cual ocurrirá siempre que la apreciación observada en el período 19 sea mayor que $E V\left(M_{19}-S_{19}, 20\right)$, más el valor esperado de no ejercer la opción ponderado por la probabilidad de no ejercer la opción (la probabilidad de que la apreciación sea menor que $\left.E V\left(M_{19}-S_{19}, 20\right)\right)$.

Siguiendo esta recursión podemos llegar fácilmente al período 1 y obtener el precio de la opción en ese período como función de $M_{o}-S_{o}$ y la volatilidad cambiaria.

Para determinar el valor de la opción, de acuerdo a la metodología planteada en este trabajo, se desarrolló un algoritmo numérico que permite establecer dicho valor en función del número remanente de períodos del contrato, del valor de la diferencia entre el logaritmo de la media móvil del tipo de cambio $(M)$ y el logaritmo de éste $(S)$ y de la volatilidad de las fluctuaciones cambiarias.

Para cada valor de la diferencia entre la media móvil y el nivel del tipo de cambio se determinó el precio de la opción en el último período. Una vez determinado el precio en este período se procedió a estimar el precio de la opción en los períodos previos, tomando como la estimación del valor en el momento $t$, el valor 
esperado de la opción en el período $t+1$.

Para esto, el primer paso fue construir una matriz de datos que albergara todas las posibles trayectorias de la diferencia entre el promedio móvil del tipo de cambio y el nivel de éste $(M-S)$, a lo largo de los 20 períodos (días) para los cuales se estima el valor esperado. Para cada nivel de $(M-S)$, se toman en cuenta sus posibles realizaciones en el período inmediato posterior en un rango de $+/-4$ desviaciones estándar, donde cada desviación estándar se halla conformada por 20 realizaciones, conformando un total de 160 posibles movimientos de $(M-S)$ en un período. De este modo se aproxima la distribución normal de la variable. ${ }^{10}$ Asimismo, se calcularon las fluctuaciones cambiarias necesarias para llevar a $(M-S)$ a cada nivel considerado en el conjunto de realizaciones posibles (veasé la ecuación 6), asignando a cada una de estas una probabilidad de ocurrencia, determinada por una distribución normal con varianza $\left(\sigma^{2}\right)$ y media igual a la depreciación promedio diaria del tipo de cambio $(\gamma)$.

Con este conjunto de posibles trayectorias de $(M-S)$, así como las probabilidades asociadas a cada evento, y dado un cierto nivel de $M-S$ en el período previo, se comenzó por resolver el valor de la opción en el último período. Partiendo del período 20, para el cual se conoce que el valor de la opción en el período siguiente será nulo (dado que ésta caduca), se calculó el valor esperado de la opción en ese período, multiplicando cada posible apreciación del tipo de cambio (80 en este caso) por la correspondiente probabilidad que le asigna su distribución normal, en el caso de que la restricción no operara $((M-S)>0)$. En los casos en que dicha restricción si operara $((M-S)<0)$ se asignó un valor de 0 . En este punto, el resultado fue una trayectoria del valor esperado de la opción en el período 19, en términos del valor de $(M-S)$ que describe dicho valor de la opción como el monto de la apreciación esperada, en el caso en que $(M-S)>0$ y de 0 en el caso contrario. En las gráficas de las páginas siguientes este valor se indica como $E V 19^{11}$.

Siguiendo el proceso recursivo, se obtuvo el valor de la opción para el período 18. Esto se realizó comparando a la apreciación correspondiente a cada realización posible de $\left(\Delta S_{t}\right)$ en el período 19 con el valor esperado de la opción en ese período y para ese nivel de $M-S$ o sea el valor esperado de no ejercer. El valor esperado de la opción para el período 18 resultó ser la suma de los productos de todas las apreciaciones mayores al valor esperado de conservar la opción al período 19 por su probabilidad de ocurrencia; más la suma de los productos del valor esperado de la opción en el período 19 y las probabilidades correspondientes a las apreciaciones menores a dicho valor esperado posterior, en caso de que la restricción

\footnotetext{
${ }^{10}$ Se supuso que este rango era suficiente para aproximar la distribución normal del tipo de cambio y contener en el casi la totalidad de las posibles observaciones aleatorias siguientes.

${ }^{11}$ El incluir una tasa de descuento no altera los resultados.
} 
no operara. En el caso de que si operara la restricción, el valor de la opción en el período 18, consistió en el valor esperado de la opción, en el período 19. En otras palabras, a la suma de los productos de los valores esperados de la opción en el período 19 y sus probabilidades asociadas.

Continuando con el proceso recursivo descrito de manera parcial anteriormente, se llegó al valor de la opción en el período 0 , en el cual, a cada valor de $(M-S)$ inicial, corresponden 3,200 realizaciones de esta variable en el período 20, cuyos valores esperados asociados se hallan resumidos en el período 0. Esta estructura se conformó de este modo ya que cada una de las realizaciones de $(M-S)$ en un período, corresponden otras 160 posibles en el siguiente período, dando lugar a que cada valor inicial de dicha diferencia, tenga un numero considerable de posibles valores finales en el período 20. La estructura anterior dio lugar a una serie de valores de la opción por cada período en función del valor de $(M-S)$.

A continuación se presentan los resultados de esta simulación, que se realiza para los siguientes valores de la volatilidad cambiaria $\left(\sigma_{\Delta s}=0.38 \%, 0.60 \%\right.$ y $0.80 \%)^{12}$. Presentamos el valor que toma la opción en cada período (del 0 al 19) como función de $M-S$ en el período anterior.

En las gráficas siguientes se muestra el valor que toma la opción en cada período ( 0 al 19) en función de la diferencia porcentual entre el nivel de tipo de cambio y el promedio móvil. Estas gráficas se realizan para diferentes niveles de volatilidad cambiaria. Los valores para los períodos 0 y 19 se indican con la abreviación $E V 0$ y $E V 19$, respectivamente.

\footnotetext{
${ }^{12}$ Hay que recordar que la volatilidad de $M-S$ es igual a la volatilidad de las fluctuaciones cambiarias multiplicada por el parámetro asociado a $\Delta S_{t}$ en la ecuación que explica el proceso seguido por $M_{t}-S_{t}(\beta=0.96)$.
} 
Desviación Estándar del Tipo de Cambio - 6\%

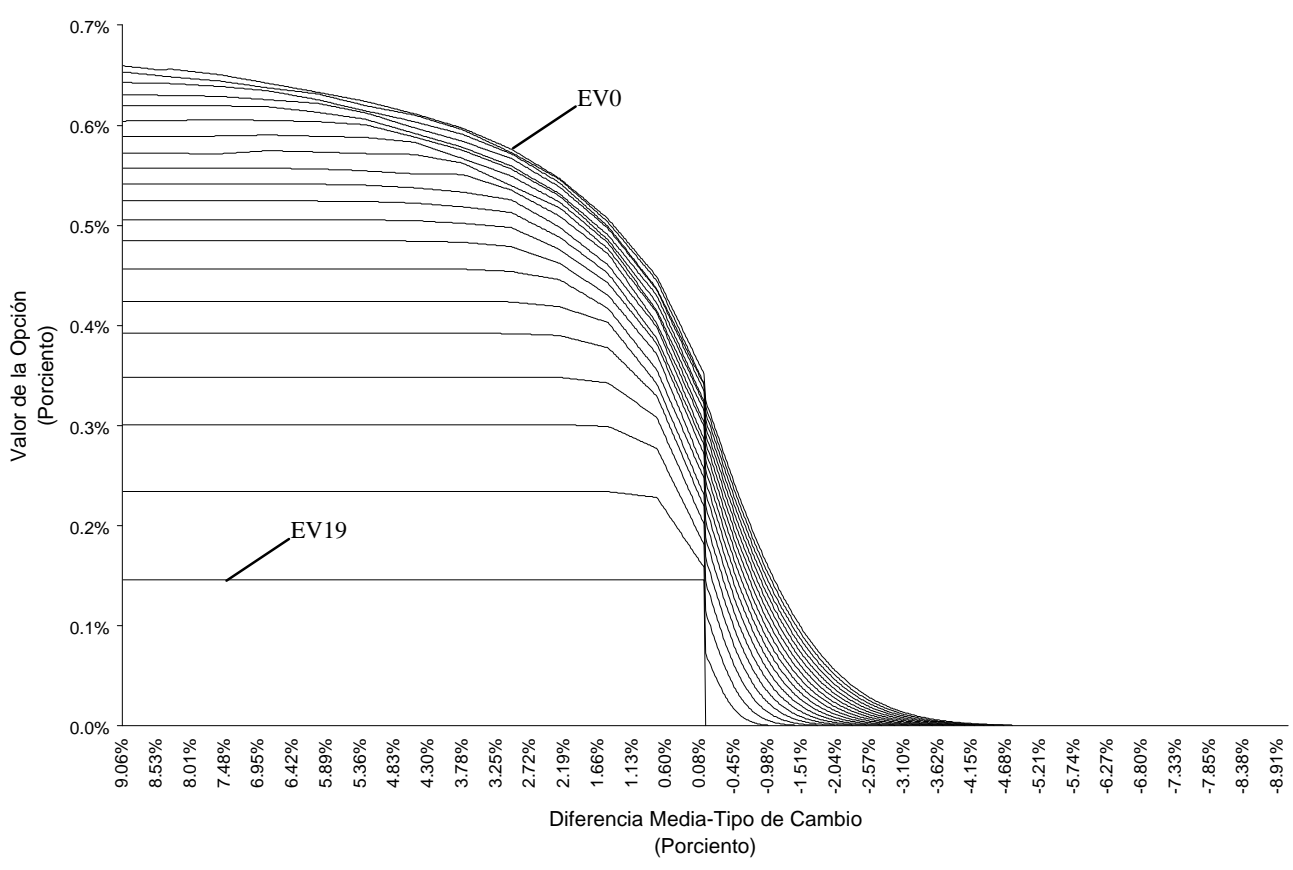

Valor de la Opción

Desviación Estándar del Tipo de Cambio - 9.5\%

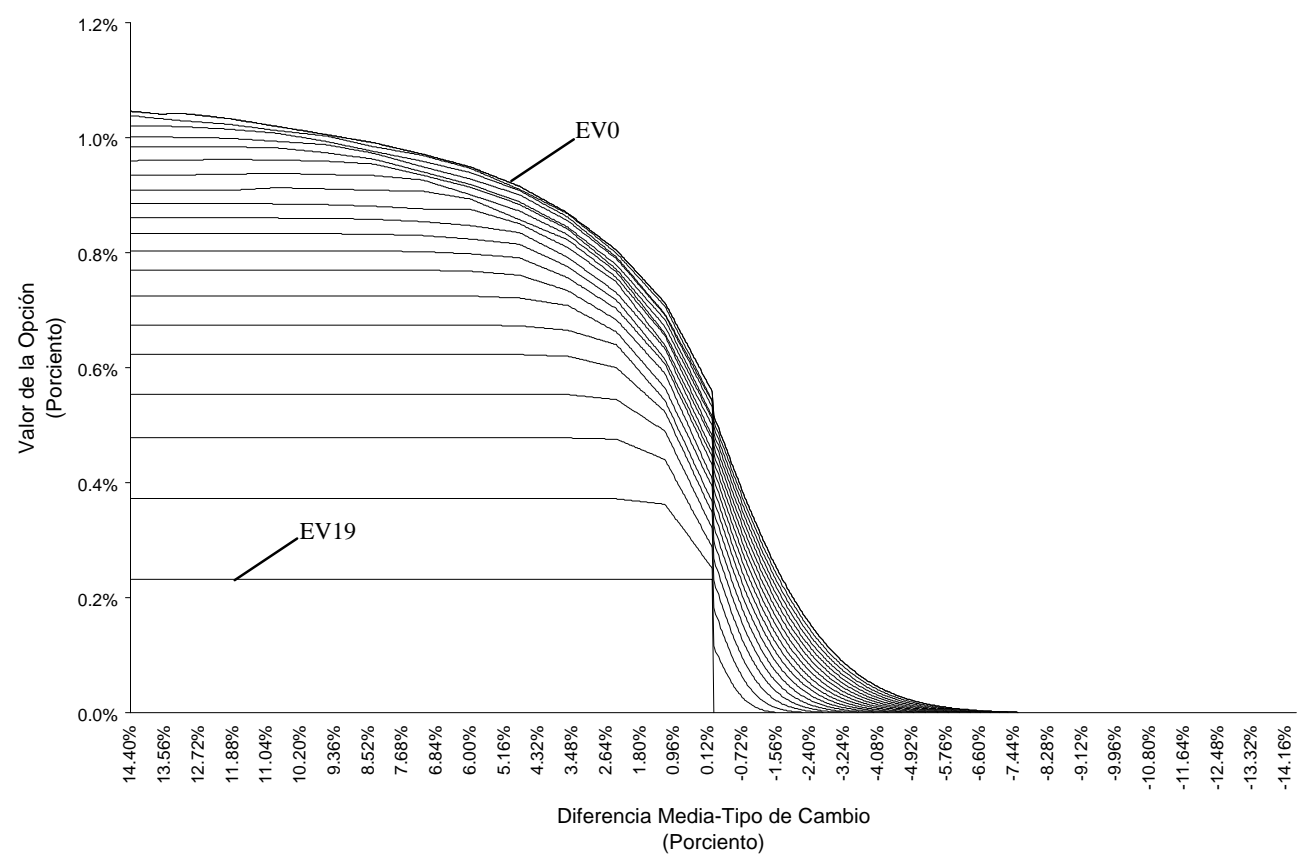




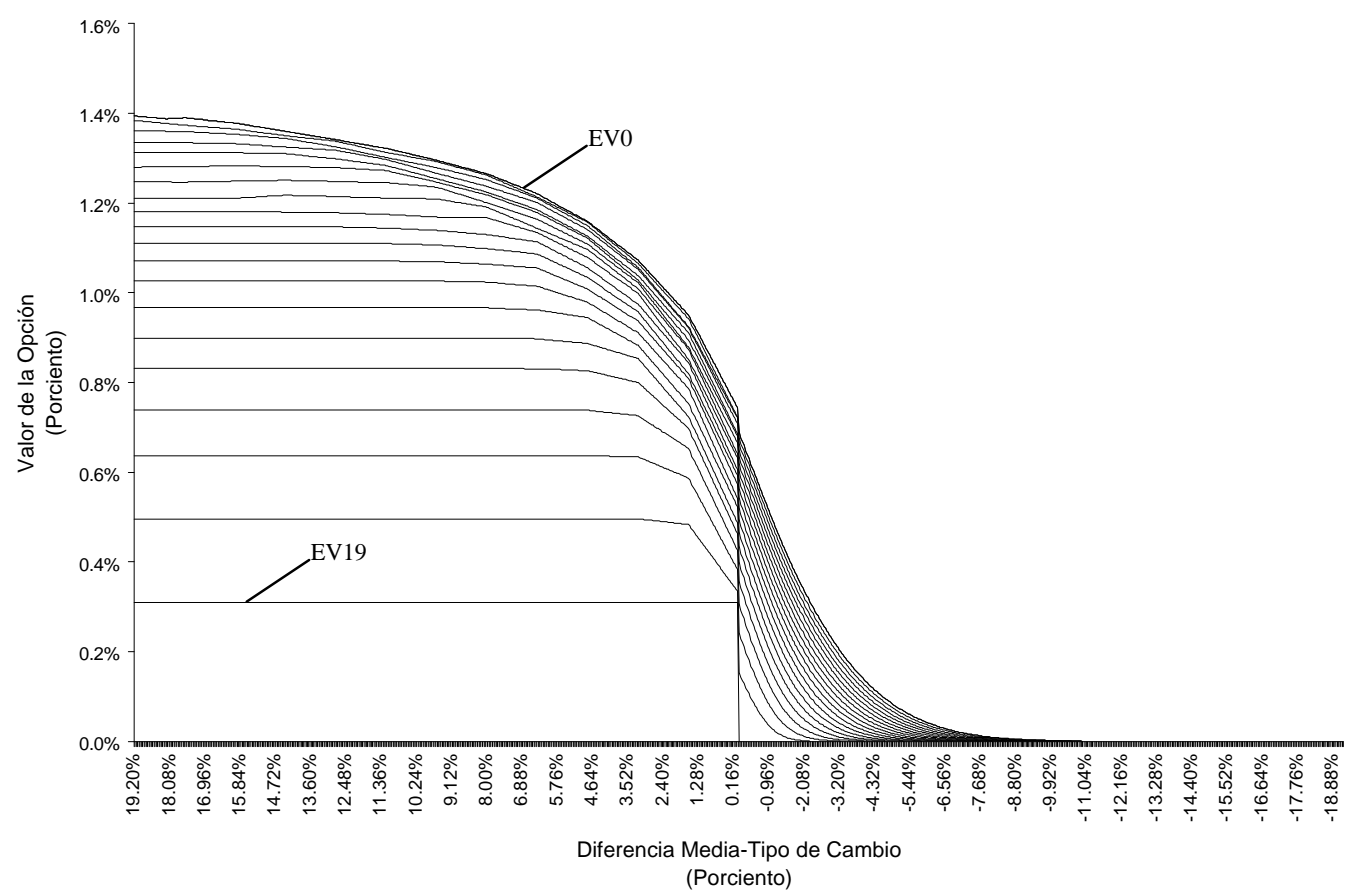

Para estudiar la sensibilidad del precio de la opción a cambios en la tendencia y volatilidad del tipo de cambio, estas simulaciones se hicieron para diferentes niveles de depreciación esperada y volatilidad del tipo de cambio $\left(\gamma\right.$ y $\left.\sigma_{\Delta s}\right)$. Se eligieron valores cercanos a la tendencia y volatilidad cambiaria observadas durante 1997. Los resultados de dichas simulaciones se presentan en el cuadro 4. En este cuadro presentamos el valor de la opción como función de la volatilidad y la tendencia del tipo de cambio y de la diferencia entre el promedio móvil y el nivel del tipo de cambio. 
Cuadro 4: Valor de la Opción en el Período Inicial

\begin{tabular}{cccc}
\hline \multicolumn{4}{c}{$\gamma=\mathbf{0} \%$} \\
\hline $\mathbf{M - S}$ & $\sigma=6 \%$ & $\sigma=9.5 \%$ & $\sigma=12.5 \%$ \\
\hline $\mathbf{- 8 . 0} \%$ & $0.000 \%$ & $0.001 \%$ & $0.010 \%$ \\
$\mathbf{- 6 . 0} \%$ & $0.000 \%$ & $0.007 \%$ & $0.041 \%$ \\
$\mathbf{- 4 . 0} \%$ & $0.003 \%$ & $0.051 \%$ & $0.134 \%$ \\
$\mathbf{- 2 . 0} \%$ & $0.057 \%$ & $0.203 \%$ & $0.348 \%$ \\
$\mathbf{0 . 0} \%$ & $0.326 \%$ & $0.518 \%$ & $0.690 \%$ \\
$\mathbf{0 . 2} \%$ & $0.357 \%$ & $0.547 \%$ & $0.723 \%$ \\
$\mathbf{0 . 4} \%$ & $0.390 \%$ & $0.581 \%$ & $0.755 \%$ \\
$\mathbf{0 . 6} \%$ & $0.421 \%$ & $0.615 \%$ & $0.788 \%$ \\
$\mathbf{1 . 0} \%$ & $0.466 \%$ & $0.679 \%$ & $0.853 \%$ \\
$\mathbf{2 . 0 \%}$ & $0.532 \%$ & $0.773 \%$ & $0.981 \%$ \\
\hline
\end{tabular}

\begin{tabular}{cccc}
\multicolumn{4}{c}{$\gamma=\mathbf{4} \%$} \\
\hline $\mathbf{M - S}$ & $\sigma=6 \%$ & $\sigma=9.5 \%$ & $\sigma=12.5 \%$ \\
\hline $\mathbf{- 8 . 0} \%$ & $0.000 \%$ & $0.001 \%$ & $0.010 \%$ \\
$\mathbf{- 6 . 0} \%$ & $0.000 \%$ & $0.007 \%$ & $0.040 \%$ \\
$\mathbf{- 4 . 0} \%$ & $0.003 \%$ & $0.046 \%$ & $0.131 \%$ \\
$\mathbf{- 2 . 0} \%$ & $0.054 \%$ & $0.188 \%$ & $0.338 \%$ \\
$\mathbf{0 . 0} \%$ & $0.308 \%$ & $0.499 \%$ & $0.672 \%$ \\
$\mathbf{0 . 2} \%$ & $0.354 \%$ & $0.560 \%$ & $0.747 \%$ \\
$\mathbf{0 . 4} \%$ & $0.383 \%$ & $0.581 \%$ & $0.778 \%$ \\
$\mathbf{0 . 6} \%$ & $0.406 \%$ & $0.610 \%$ & $0.794 \%$ \\
$\mathbf{1 . 0} \%$ & $0.443 \%$ & $0.667 \%$ & $0.852 \%$ \\
$\mathbf{2 . 0 \%}$ & $0.509 \%$ & $0.749 \%$ & $0.958 \%$ \\
\hline
\end{tabular}

\begin{tabular}{cccc}
\multicolumn{4}{c}{$\gamma=\mathbf{8} \%$} \\
\hline $\mathbf{M}-\mathrm{S}$ & $\sigma=6 \%$ & $\sigma=9.5 \%$ & $\sigma=12.5 \%$ \\
\hline $\mathbf{- 8 . 0} \%$ & $0.000 \%$ & $0.001 \%$ & $0.009 \%$ \\
$\mathbf{- 6 . 0} \%$ & $0.000 \%$ & $0.007 \%$ & $0.039 \%$ \\
$\mathbf{- 4 . 0} \%$ & $0.003 \%$ & $0.044 \%$ & $0.127 \%$ \\
$\mathbf{- 2 . 0} \%$ & $0.048 \%$ & $0.182 \%$ & $0.330 \%$ \\
$\mathbf{0 . 0} \%$ & $0.276 \%$ & $0.483 \%$ & $0.656 \%$ \\
$\mathbf{0 . 2} \%$ & $0.317 \%$ & $0.539 \%$ & $0.731 \%$ \\
$\mathbf{0 . 4} \%$ & $0.345 \%$ & $0.571 \%$ & $0.753 \%$ \\
$\mathbf{0 . 6} \%$ & $0.366 \%$ & $0.590 \%$ & $0.771 \%$ \\
$\mathbf{1 . 0} \%$ & $0.400 \%$ & $0.644 \%$ & $0.828 \%$ \\
$\mathbf{2 . 0} \%$ & $0.463 \%$ & $0.729 \%$ & $0.934 \%$ \\
\hline
\end{tabular}

\begin{tabular}{cccc}
\multicolumn{4}{c}{$\gamma=\mathbf{1 2} \%$} \\
\hline $\mathbf{M}-\mathbf{S}$ & $\sigma=6 \%$ & $\sigma=9.5 \%$ & $\sigma=12.5 \%$ \\
\hline $\mathbf{- 8 . 0} \%$ & $0.000 \%$ & $0.001 \%$ & $0.009 \%$ \\
$\mathbf{- 6 . 0} \%$ & $0.000 \%$ & $0.007 \%$ & $0.038 \%$ \\
$\mathbf{- 4 . 0} \%$ & $0.003 \%$ & $0.042 \%$ & $0.124 \%$ \\
$\mathbf{- 2 . 0} \%$ & $0.004 \%$ & $0.175 \%$ & $0.321 \%$ \\
$\mathbf{0 . 0} \%$ & $0.266 \%$ & $0.466 \%$ & $0.638 \%$ \\
$\mathbf{0 . 2} \%$ & $0.301 \%$ & $0.521 \%$ & $0.707 \%$ \\
$\mathbf{0 . 4} \%$ & $0.325 \%$ & $0.548 \%$ & $0.729 \%$ \\
$\mathbf{0 . 6} \%$ & $0.334 \%$ & $0.569 \%$ & $0.755 \%$ \\
$\mathbf{1 . 0} \%$ & $0.389 \%$ & $0.619 \%$ & $0.816 \%$ \\
$\mathbf{2 . 0} \%$ & $0.423 \%$ & $0.711 \%$ & $0.920 \%$ \\
\hline
\end{tabular}


En estas gráficas y cuadros se aprecian varios resultados, entre los que destacan los siguientes:

i) A mayor volatilidad de las fluctuaciones cambiarias, mayor será el precio de la opción. Esto se observa tanto en la Gráfica 2 como en el Cuadro 4, ya que a medida que la volatilidad cambiaria aumenta, para la misma desviación porcentual entre la media móvil del tipo de cambio y el nivel de éste, corresponde un valor mayor de la opción en todos los períodos. Este resultado se explica por el hecho de que al aumentar la volatilidad cambiaria, la probabilidad de mayores apreciaciones aumenta, lo cual incrementa la ganancia esperada de ejercer la opción.

ii) El valor de la opción es creciente en las diferencias entre el promedio móvil y el nivel del tipo de cambio, lo cual ocurre a tasas de crecimiento decrecientes. Este resultado es muy intuitivo, ya que mientras mayor sea esta diferencia, menor será la probabilidad de que la restricción sea efectiva en el futuro y la opción no se pueda ejercer. Esto ocurre a tasas decrecientes, ya que una vez que la diferencia entre el promedio móvil y el tipo de cambio es grande, la probabilidad de que se cumpla la restricción en los próximos 20 períodos es muy pequeña. Debido a esto, aumentos en la diferencia entre el promedio móvil y el tipo de cambio ya no afectarán significativamente dicha probabilidad.

iii) De las gráficas también podemos deducir la función de ejercicio de la opción. En la página 11 se estableció que la opción se ejerce en el período $t$, siempre que la apreciación en dicho período sea mayor que el valor esperado de la opción en ese período, la cual refleja el valor de esperarse hasta el siguiente período y que la restricción no se cumpla. Por lo tanto, en las gráficas, la función del valor de la opción en cada período nos da el nivel mínimo de apreciación que se debe observar para que se ejerza la opción, suponiendo que $M-S>0$.

Debido a esto, se observa que al aumentar la volatilidad cambiaria aumentan los niveles de apreciación necesarios para que se ejerza la opción en cada período. Esto se debe a que al aumentar el valor de esperarse, los dueños de la opción se vuelven más exigentes en su decisión de ejercicio.

iv) También se observa que el valor de la opción disminuye a medida que el término de la opción se acerca, lo cual ocurre a tasas crecientes. De estas observaciones también se deriva que, a partir del período 15, el aumento en el valor de la opción al aumentar en un día la vigencia de la opción, es muy pequeño.

v) El valor de la opción en cada período presenta un salto discreto cuando la diferencia entre el promedio móvil y el tipo de cambio es 0 . Esto refleja el valor que 
se pierde por no poder ejercer la opción en ese día, debido a que se cumple la restricción. La magnitud de esta discontinuidad es mayor mientras menos períodos de vida le quedan a la opción.

vi) El valor de la opción disminuye al aumentar la tendencia hacia la depreciación del proceso seguido por el tipo de cambio; medida por el parámetro $\gamma$. Este resultado es bastante obvio ya que al aumentar la tendencia del tipo de cambio las posibles apreciaciones son menores y por lo tanto disminuye el valor de la opción.

Dado que del análisis anterior se obtiene la función de ejercicio óptimo, se pueden realizar simulaciones de la trayectoria que sigue el tipo de cambio y el promedio móvil durante la vigencia de la opción y determinar, de acuerdo a la función de ejercicio, el momento de ejercicio de la opción y la ganancia que se obtiene.

Para evaluar el efecto de esta política sobre la frecuencia y el momento de las intervenciones se realizaron diversas simulaciones del proceso seguido por el tipo de cambio y se estableció el momento de ejercicio de la opción. Para cada combinación de volatilidad y tendencia cambiaria se tomaron 10 valores iniciales de la diferencia entre la media móvil de tipo de cambio y su nivel, y para cada uno de ellos se realizaron 1,000 simulaciones de la trayectoria que siguen el tipo de cambio y su media móvil durante el mes. Con base en la función de ejercicio derivada anteriormente se registró, para cada simulación, el período de ejercicio y la apreciación observada en ese período. También se realizó un ejercicio para evaluar, dada la tendencia y volatilidad de las fluctuaciones cambiarias, el valor esperado del número de veces que las opciones son ejercidas en el año. Este ejercicio se realizó suponiendo que las opciones se subastan una vez por mes, por lo tanto el valor máximo será 12 veces y el mínimo 0 . Por ejemplo, para $\sigma=0.38 \%$ y $\gamma=0$, vemos que cuando $M-S=0$, la probabilidad de ejercicio en el mes será de $88.1 \%$ y durante el año se ejercerán el $82.1 \%$ de las opciones subastadas. También se puede apreciar que si al momento de subastarse $M-S=0$, el período promedio de ejercicio es de 5.7 días. En el Cuadro 5 se muestran los resultados de estos ejercicios. 


\section{Cuadro 5}

$$
\gamma=0 \%
$$

\begin{tabular}{|c|c|c|c|c|c|c|c|c|c|c|c|}
\hline \multirow[b]{3}{*}{ M-S } & \multicolumn{3}{|c|}{$\sigma=6 \%$} & \multicolumn{4}{|c|}{$\sigma=9.5 \%$} & \multirow[b]{3}{*}{ M-S } & \multicolumn{3}{|c|}{$\sigma=12.5 \%$} \\
\hline & \multirow[b]{2}{*}{ Periodo } & \multicolumn{2}{|c|}{ Ejercicio } & \multirow[b]{2}{*}{ M-S } & \multirow[b]{2}{*}{ Periodo } & \multicolumn{2}{|c|}{ Ejercicio } & & \multirow[b]{2}{*}{ Periodo } & \multicolumn{2}{|c|}{ Ejercicio } \\
\hline & & Mensual & Anual & & & Mensual & Anual & & & Mensual & Anual \\
\hline$-8.0 \%$ & 18.5 & $5.30 \%$ & $75.24 \%$ & $-8.0 \%$ & 17.1 & $20.30 \%$ & $76.78 \%$ & $-8.0 \%$ & 15.8 & $32.60 \%$ & $78.00 \%$ \\
\hline$-6.0 \%$ & 17.3 & $16.40 \%$ & $76.34 \%$ & $-6.0 \%$ & 15.5 & $32.50 \%$ & $78.06 \%$ & $-6.0 \%$ & 14.5 & $46.90 \%$ & $80.15 \%$ \\
\hline$-4.0 \%$ & 16.3 & $30.10 \%$ & $78.00 \%$ & $-4.0 \%$ & 13.7 & $50.20 \%$ & $79.08 \%$ & $-4.0 \%$ & 12.9 & $57.60 \%$ & $82.04 \%$ \\
\hline$-2.0 \%$ & 12.5 & $55.10 \%$ & $79.99 \%$ & $-2.0 \%$ & 10.5 & $67.20 \%$ & $81.00 \%$ & $-2.0 \%$ & 9.4 & $72.70 \%$ & $84.63 \%$ \\
\hline $0.0 \%$ & 5.7 & $88.00 \%$ & $82.13 \%$ & $0.0 \%$ & 6.0 & $87.80 \%$ & $83.89 \%$ & $0.0 \%$ & 6.1 & $87.00 \%$ & $86.45 \%$ \\
\hline $0.2 \%$ & 5.3 & $89.80 \%$ & $85.88 \%$ & $0.2 \%$ & 6.0 & $89.30 \%$ & $86.62 \%$ & $0.2 \%$ & 6.0 & $88.10 \%$ & $88.24 \%$ \\
\hline $0.4 \%$ & 5.5 & $90.00 \%$ & $88.65 \%$ & $0.4 \%$ & 5.9 & $89.60 \%$ & $88.63 \%$ & $0.4 \%$ & 5.9 & $89.40 \%$ & $90.25 \%$ \\
\hline $0.6 \%$ & 5.9 & $92.20 \%$ & $90.02 \%$ & $0.6 \%$ & 6.0 & $90.90 \%$ & $91.05 \%$ & $0.6 \%$ & 5.7 & $90.10 \%$ & $92.14 \%$ \\
\hline $1.0 \%$ & 5.4 & $93.20 \%$ & $93.95 \%$ & $1.0 \%$ & 6.0 & $92.90 \%$ & $93.48 \%$ & $1.0 \%$ & 5.5 & $92.30 \%$ & $94.05 \%$ \\
\hline $2.0 \%$ & 5.1 & $98.90 \%$ & $98.88 \%$ & $2.0 \%$ & 5.5 & $97.50 \%$ & $97.95 \%$ & $2.0 \%$ & 4.9 & $97.10 \%$ & $97.66 \%$ \\
\hline
\end{tabular}

\begin{tabular}{|c|c|c|c|c|c|c|c|c|c|c|c|}
\hline \multirow[b]{3}{*}{ M-S } & \multicolumn{3}{|c|}{$\sigma=6 \%$} & \multirow[b]{3}{*}{ M-S } & \multirow[b]{3}{*}{ Periodo } & \multicolumn{2}{|l|}{$\sigma=9.5 \%$} & \multirow[b]{3}{*}{ M-S } & \multicolumn{3}{|c|}{$\sigma=12.5 \%$} \\
\hline & \multirow[b]{2}{*}{ Periodo } & \multicolumn{2}{|c|}{ Ejercicio } & & & Ejerc & & & & Ejer & \\
\hline & & Mensual & Anual & & & Mensual & Anual & & Periodo & Mensual & Anual \\
\hline$-8.0 \%$ & 18.3 & $3.80 \%$ & $72.83 \%$ & $-8.0 \%$ & 16.9 & $17.10 \%$ & $73.90 \%$ & $-8.0 \%$ & 15.1 & $27.40 \%$ & $74.08 \%$ \\
\hline$-6.0 \%$ & 17.6 & $12.40 \%$ & $74.25 \%$ & $-6.0 \%$ & 15.8 & $28.80 \%$ & $75.91 \%$ & $-6.0 \%$ & 14.0 & $36.90 \%$ & $76.75 \%$ \\
\hline$-4.0 \%$ & 15.9 & $25.40 \%$ & $76.20 \%$ & $-4.0 \%$ & 14.0 & $45.10 \%$ & $77.66 \%$ & $-4.0 \%$ & 12.6 & $52.60 \%$ & $79.16 \%$ \\
\hline$-2.0 \%$ & 13.3 & $50.30 \%$ & $77.75 \%$ & $-2.0 \%$ & 11.1 & $62.00 \%$ & $79.58 \%$ & $-2.0 \%$ & 9.7 & $66.10 \%$ & $81.75 \%$ \\
\hline $0.0 \%$ & 5.0 & $86.30 \%$ & $79.79 \%$ & $0.0 \%$ & 6.0 & $85.70 \%$ & $82.33 \%$ & $0.0 \%$ & 5.9 & $85.80 \%$ & $81.89 \%$ \\
\hline $0.2 \%$ & 4.8 & $88.80 \%$ & $83.25 \%$ & $0.2 \%$ & 5.9 & $88.20 \%$ & $83.66 \%$ & $0.2 \%$ & 5.7 & $87.10 \%$ & $82.41 \%$ \\
\hline $0.4 \%$ & 4.3 & $91.20 \%$ & $86.62 \%$ & $0.4 \%$ & 5.8 & $89.01 \%$ & $85.39 \%$ & $0.4 \%$ & 6.0 & $89.80 \%$ & $84.20 \%$ \\
\hline $0.6 \%$ & 4.0 & $93.70 \%$ & $88.37 \%$ & $0.6 \%$ & 5.7 & $90.80 \%$ & $86.90 \%$ & $0.6 \%$ & 5.7 & $90.30 \%$ & $86.00 \%$ \\
\hline $1.0 \%$ & 4.2 & $95.10 \%$ & $91.20 \%$ & $1.0 \%$ & 6.1 & $94.50 \%$ & $90.10 \%$ & $1.0 \%$ & 5.6 & $92.00 \%$ & $89.90 \%$ \\
\hline $2.0 \%$ & 4.2 & $99.01 \%$ & $95.80 \%$ & $2.0 \%$ & 6.0 & $97.50 \%$ & $95.00 \%$ & $2.0 \%$ & 5.2 & $96.30 \%$ & $94.70 \%$ \\
\hline
\end{tabular}

\begin{tabular}{|c|c|c|c|c|c|c|c|c|c|c|c|}
\hline \multirow[b]{3}{*}{ M-S } & \multicolumn{3}{|c|}{$\sigma=6 \%$} & \multirow[b]{3}{*}{ M-S } & \multicolumn{3}{|c|}{$\sigma=9.5 \%$} & \multirow[b]{3}{*}{ M-S } & \multicolumn{3}{|c|}{$\sigma=12.5 \%$} \\
\hline & \multirow[b]{2}{*}{ Periodo } & \multicolumn{2}{|c|}{ Ejercicio } & & \multirow[b]{2}{*}{ Periodo } & \multicolumn{2}{|c|}{ Ejercicio } & & \multirow[b]{2}{*}{ Periodo } & \multicolumn{2}{|c|}{ Ejercicio } \\
\hline & & Mensual & Anual & & & Mensual & Anual & & & Mensual & Anual \\
\hline$-8.0 \%$ & 18.4 & $2.90 \%$ & $62.83 \%$ & $-8.0 \%$ & 15.8 & $13.00 \%$ & $69.33 \%$ & $-8.0 \%$ & 15.6 & $26.30 \%$ & $72.75 \%$ \\
\hline$-6.0 \%$ & 17.4 & $6.70 \%$ & $65.58 \%$ & $-6.0 \%$ & 15.3 & $21.10 \%$ & $70.91 \%$ & $-6.0 \%$ & 14.3 & $34.10 \%$ & $75.00 \%$ \\
\hline$-4.0 \%$ & 16.3 & $20.00 \%$ & $68.58 \%$ & $-4.0 \%$ & 13.8 & $34.60 \%$ & $72.91 \%$ & $-4.0 \%$ & 12.6 & $51.30 \%$ & $78.50 \%$ \\
\hline$-2.0 \%$ & 13.0 & $46.40 \%$ & $71.42 \%$ & $-2.0 \%$ & 10.3 & $55.70 \%$ & $74.91 \%$ & $-2.0 \%$ & 9.7 & $65.30 \%$ & $78.75 \%$ \\
\hline $0.0 \%$ & 5.9 & $79.80 \%$ & $75.08 \%$ & $0.0 \%$ & 5.6 & $82.20 \%$ & $78.66 \%$ & $0.0 \%$ & 6.0 & $85.80 \%$ & $80.09 \%$ \\
\hline $0.2 \%$ & 5.7 & $83.60 \%$ & $75.41 \%$ & $0.2 \%$ & 5.7 & $85.80 \%$ & $80.02 \%$ & $0.2 \%$ & 5.8 & $86.15 \%$ & $82.10 \%$ \\
\hline $0.4 \%$ & 5.9 & $84.80 \%$ & $77.41 \%$ & $0.4 \%$ & 5.6 & $86.80 \%$ & $81.80 \%$ & $0.4 \%$ & 5.4 & $88.88 \%$ & $88.58 \%$ \\
\hline $0.6 \%$ & 5.8 & $86.90 \%$ & $80.48 \%$ & $0.6 \%$ & 5.5 & $87.60 \%$ & $85.60 \%$ & $0.6 \%$ & 5.4 & $92.00 \%$ & $89.01 \%$ \\
\hline $1.0 \%$ & 5.1 & $90.80 \%$ & $85.59 \%$ & $1.0 \%$ & 5.3 & $89.00 \%$ & $91.70 \%$ & $1.0 \%$ & 5.3 & $92.20 \%$ & $94.05 \%$ \\
\hline $2.0 \%$ & 5.0 & $98.80 \%$ & $96.91 \%$ & $2.0 \%$ & 4.8 & $97.50 \%$ & $96.10 \%$ & $2.0 \%$ & 5.0 & $98.30 \%$ & $95.60 \%$ \\
\hline
\end{tabular}

$\gamma=12 \%$

\begin{tabular}{|c|c|c|c|c|c|c|c|c|c|c|c|}
\hline \multirow[b]{3}{*}{ M-S } & \multicolumn{3}{|c|}{$\sigma=6 \%$} & \multicolumn{4}{|c|}{$\sigma=9.5 \%$} & \multirow[b]{3}{*}{ M-S } & \multicolumn{3}{|c|}{$\sigma=12.5 \%$} \\
\hline & \multirow[b]{2}{*}{ Periodo } & \multicolumn{2}{|c|}{ Ejercicio } & \multirow[b]{2}{*}{ M-S } & \multirow[b]{2}{*}{ Periodo } & \multicolumn{2}{|c|}{ Ejercicio } & & \multirow[b]{2}{*}{ Periodo } & \multicolumn{2}{|c|}{ Ejercicio } \\
\hline & & Mensual & Anual & & & Mensual & Anual & & & Mensual & Anual \\
\hline$-8.0 \%$ & 17.8 & $1.70 \%$ & $58.00 \%$ & $-8.0 \%$ & 17.4 & $13.80 \%$ & $68.10 \%$ & $-8.0 \%$ & 16.0 & $24.40 \%$ & $72.22 \%$ \\
\hline$-6.0 \%$ & 18.4 & $5.10 \%$ & $57.58 \%$ & $-6.0 \%$ & 15.8 & $22.60 \%$ & $67.79 \%$ & $-6.0 \%$ & 14.7 & $33.30 \%$ & $74.08 \%$ \\
\hline$-4.0 \%$ & 16.5 & $15.90 \%$ & $61.33 \%$ & $-4.0 \%$ & 14.0 & $37.00 \%$ & $69.41 \%$ & $-4.0 \%$ & 12.5 & $47.80 \%$ & $75.00 \%$ \\
\hline$-2.0 \%$ & 13.2 & $36.90 \%$ & $62.66 \%$ & $-2.0 \%$ & 10.8 & $56.20 \%$ & $72.66 \%$ & $-2.0 \%$ & 9.8 & $65.20 \%$ & $74.00 \%$ \\
\hline $0.0 \%$ & 5.8 & $78.30 \%$ & $67.40 \%$ & $0.0 \%$ & 6.1 & $83.30 \%$ & $78.00 \%$ & $0.0 \%$ & 6.2 & $85.60 \%$ & $78.10 \%$ \\
\hline $0.2 \%$ & 5.8 & $83.70 \%$ & $68.00 \%$ & $0.2 \%$ & 6.0 & $85.80 \%$ & $76.79 \%$ & $0.2 \%$ & 6.0 & $86.10 \%$ & $79.20 \%$ \\
\hline $0.4 \%$ & 5.6 & $84.80 \%$ & $70.09 \%$ & $0.4 \%$ & 6.0 & $86.30 \%$ & $77.58 \%$ & $0.4 \%$ & 5.6 & $88.20 \%$ & $81.20 \%$ \\
\hline $0.6 \%$ & 5.4 & $86.70 \%$ & $72.31 \%$ & $0.6 \%$ & 5.6 & $87.80 \%$ & $79.90 \%$ & $0.6 \%$ & 5.4 & $91.20 \%$ & $82.12 \%$ \\
\hline $\begin{array}{l}1.0 \% \\
2.0 \%\end{array}$ & $\begin{array}{l}5.1 \\
4.9\end{array}$ & $\begin{array}{l}91.10 \% \\
97.80 \%\end{array}$ & $\begin{array}{l}79.24 \% \\
88.90 \%\end{array}$ & $\begin{array}{l}1.0 \% \\
2.0 \% \\
\end{array}$ & $\begin{array}{l}5.8 \\
5.2\end{array}$ & $\begin{array}{l}90.10 \% \\
96.90 \%\end{array}$ & $\begin{array}{l}81.20 \% \\
92.16 \%\end{array}$ & $\begin{array}{l}1.0 \% \\
2.0 \%\end{array}$ & $\begin{array}{l}5.3 \\
4.9 \\
\end{array}$ & $\begin{array}{l}92.10 \% \\
98.80 \%\end{array}$ & $\begin{array}{l}85.14 \% \\
92.00 \%\end{array}$ \\
\hline
\end{tabular}


De los resultados anteriores se pueden obtener las siguientes conclusiones:

i) Cuando en el período inicial opera la restricción $(M-S<0)$, vemos que al aumentar la varianza se incrementa la probabilidad de ejercicio y disminuye el período promedio de ejercicio. Este resultado se debe a que al aumentar la varianza, la probabilidad de que en algún período futuro la restricción no opere se incrementa, esto influye positivamente en la probabilidad de ejercicio. Por otro lado, cuando la restricción no opera en el período inicial y la tendencia del tipo de cambio $(\gamma)$ es baja, a mayor varianza menor probabilidad de ejercicio. Este resultado nos indica que al aumentar la volatilidad de las fluctuaciones cambiarias, los dueños de la opción se vuelven más exigentes y ejercen con apreciaciones del tipo de cambio mayores, lo que resulta en que las opciones sean ejercidas con menos frecuencia. Por otro lado, cuando la opción es ejercida, la ganancia obtenida más que compensa las pérdidas en los casos en que no fue ejercida. Hay que destacar que en la región en la cual la restricción no opera, la probabilidad de ejercicio no se ve afectada en una magnitud importante cuando cambian los parámetros del proceso seguido por el tipo de cambio $\left(\sigma_{\Delta s}\right.$ y $\left.\gamma\right)$.

ii) Cuando aumenta la tendencia hacia la depreciación del tipo de cambio y mantenemos la volatilidad constante se observa que si la restricción opera en el período inicial, disminuye la probabilidad de ejercicio; este efecto se disipa cuando nos movemos hacia valores de $(M-S)$ iniciales positivos. En este último caso, la probabilidad y el período promedio de ejercicio no se ven alterados considerablemente al aumentar la tendencia cambiaria.

iii) También, se observa que mientras mayor sea la diferencia entre el promedio móvil y el nivel del tipo de cambio, dada una varianza constante, la probabilidad de ejercicio es mayor y la opción se ejerce más temprano.

iv) $\mathrm{Al}$ analizar el porcentaje de las opciones ejercidas en el año, destaca uno de los resultados más importantes del esquema de intervención a través de opciones. Vemos que si la situación inicial de $M-S$ es positiva o cercana a cero este porcentaje se encuentra entre 70 y $95 \%$ y depende fundamentalmente del valor inicial de $M-S$ y no de los valores de $\gamma$ y $\sigma_{\Delta s}$. Este resultado nos indica que el monto de acumulación de reservas internacionales que se lograría durante 1 año, mediante este mecanismo, es independiente del proceso que sigue el tipo de cambio. Esto presenta 2 características muy importantes. Primero, si la Comisión de Cambios programa una acumulación anual de divisas, ésta se puede obtener a través de este mecanismo sin la necesidad de pronosticar de una manera adecuada la volatilidad y tendencia del tipo de cambio. En segundo lugar, dado que bajo este esquema la acumulación no depende de los parámetros que determinan el proceso del tipo de cambio, no se está 
enviando al mercado ninguna señal sobre el nivel, depreciación o volatilidad deseadas del tipo de cambio.

\section{IV.- Estimación Empírica:}

En el Cuadro 6 se compara para cada subasta de opciones realizada, la prima pagada por ellas en dicha subasta con la prima calculada según la metodología desarrollada en este trabajo. Para calcular esta prima, se tomó la desviación entre el promedio móvil y el tipo de cambio observado el día de la subasta. La tendencia del tipo de cambio se calculó a partir de la depreciación esperada implícita en las coberturas cambiarias a 1 mes y para medir la volatilidad cambiaria se tomó la volatilidad implícita en las opciones sobre los futuros del peso. El resultado de esta comparación nos indica que la prima pagada por las opciones en las subastas realizadas por el Banco de México ha estado consistentemente por debajo de los valores teóricos en aproximadamente 53.4\%. 


\section{Cuadro 6}

\begin{tabular}{|c|c|c|c|c|}
\hline $\begin{array}{c}\begin{array}{c}\text { Fecha } \\
\text { de la } \\
\text { Subasta }\end{array} \\
\end{array}$ & $\begin{array}{l}\text { Vigencia } \\
\text { de la } \\
\text { Opción } \\
\end{array}$ & $\begin{array}{c}\text { Monto } \\
\text { Subastado } \\
\text { (MDD) }\end{array}$ & $\begin{array}{c}\text { Prima } \\
\text { Ponderada } \\
(\$ \mathrm{x} \text { Mil DIs })\end{array}$ & $\begin{array}{c}\text { Prima } \\
\text { Teórica* }^{*} \\
\text { (\$ x Mil DIs) }\end{array}$ \\
\hline 7-Ago-96 & Ago-96 & 130 & $\begin{array}{c}11.68 \\
(0.16 \%)\end{array}$ & $\begin{array}{c}51.31 \\
(0.68 \%)\end{array}$ \\
\hline 30-Ago-96 & Sep-96 & 200 & $\begin{array}{c}4.65 \\
(0.06 \%)\end{array}$ & $\begin{array}{c}40.36 \\
(0.53 \%)\end{array}$ \\
\hline 30-Sep-96 & Oct-96 & 200 & $\begin{array}{c}10.18 \\
(0.13 \%)\end{array}$ & $\begin{array}{c}52.06 \\
(0.69 \%)\end{array}$ \\
\hline 31-Oct-96 & Nov-96 & 200 & $\begin{array}{c}10.32 \\
(0.13 \%)\end{array}$ & $\begin{array}{c}46.68 \\
(0.58 \%)\end{array}$ \\
\hline 29-Nov-96 & Dic-96 & 200 & $\begin{array}{c}14.43 \\
(0.18 \%)\end{array}$ & $\begin{array}{c}36.54 \\
(0.46 \%)\end{array}$ \\
\hline 30-Dic-96 & Ene-97 & 300 & $\begin{array}{c}13.75 \\
(0.18 \%)\end{array}$ & $\begin{array}{c}32.33 \\
(0.41 \%)\end{array}$ \\
\hline 31-Ene-96 & Feb-97 & 300 & $\begin{array}{c}16.14 \\
(0.21 \%)\end{array}$ & $\begin{array}{c}41.94 \\
(0.57 \%)\end{array}$ \\
\hline 21-Feb-97 & $\begin{array}{c}\text { Feb-97 } \\
\text { (segunda) }\end{array}$ & 300 & $\begin{array}{c}15.08 \\
(0.19 \%)\end{array}$ & $\begin{array}{c}30.15 \\
(0.39 \%)\end{array}$ \\
\hline 28-Feb-97 & Mar-97 & 300 & $\begin{array}{c}10.4 \\
(0.13 \%)\end{array}$ & $\begin{array}{c}19.95 \\
(0.25 \%)\end{array}$ \\
\hline 31-Mar-97 & Abr-97 & 300 & $\begin{array}{c}21.86 \\
(0.28 \%)\end{array}$ & $\begin{array}{c}37.09 \\
(0.47 \%)\end{array}$ \\
\hline 15-Abr-97 & $\begin{array}{c}\text { Abr-97 } \\
\text { (segunda) }\end{array}$ & 300 & $\begin{array}{c}13.86 \\
(0.18 \%)\end{array}$ & $\begin{array}{c}26.76 \\
(0.34 \%)\end{array}$ \\
\hline 30-Abr-97 & May-97 & 300 & $\begin{array}{c}14.38 \\
(0.18 \%)\end{array}$ & $\begin{array}{c}32.51 \\
(0.41 \%)\end{array}$ \\
\hline 30-May-97 & Jun-97 & 300 & $\begin{array}{c}11.74 \\
(0.15 \%)\end{array}$ & $\begin{array}{c}32.65 \\
(0.41 \%)\end{array}$ \\
\hline 30-Jun-97 & Jul-97 & 300 & $\begin{array}{c}20.61 \\
(0.26 \%)\end{array}$ & $\begin{array}{c}39.23 \\
(0.49 \%)\end{array}$ \\
\hline 9-Jul-97 & $\begin{array}{c}\text { Jul-97 } \\
\text { (segunda) }\end{array}$ & 300 & $\begin{array}{c}19.57 \\
(0.25 \%)\end{array}$ & $\begin{array}{c}32.99 \\
(0.42 \%)\end{array}$ \\
\hline 31-Jul-97 & Ago-97 & 500 & $\begin{array}{c}23.36 \\
(0.30 \%)\end{array}$ & $\begin{array}{c}37.55 \\
(0.48 \%)\end{array}$ \\
\hline 29-Ago-97 & Sep-97 & 500 & $\begin{array}{c}12.27 \\
(0.16 \%)\end{array}$ & $\begin{array}{c}24.13 \\
(0.31 \%)\end{array}$ \\
\hline 30-Sep-97 & Oct-97 & 400 & $\begin{array}{c}16.10 \\
(0.21 \%)\end{array}$ & $\begin{array}{c}20.07 \\
(0.26 \%)\end{array}$ \\
\hline 31-Oct-97 & Nov-97 & 250 & $\begin{array}{c}13.05 \\
(0.16 \%)\end{array}$ & $\begin{array}{c}42.68 \\
(0.51 \%)\end{array}$ \\
\hline 28-Nov-97 & Dic-97 & 250 & $\begin{array}{c}30.05 \\
(0.37 \%)\end{array}$ & $\begin{array}{c}72.85 \\
(0.89 \%)\end{array}$ \\
\hline 15-Dic-97 & Dic-97 & 250 & $\begin{array}{c}33.81 \\
(0.42 \%)\end{array}$ & $\begin{array}{c}54.93 \\
(0.68 \%)\end{array}$ \\
\hline 30-Dic-97 & Ene-97 & 250 & $\begin{array}{c}33.54 \\
(0.42 \%)\end{array}$ & $\begin{array}{c}40.31 \\
(0.50 \%)\end{array}$ \\
\hline 30-Ene-98 & Feb-98 & 250 & $\begin{array}{c}15.85 \\
(0.19 \%)\end{array}$ & $\begin{array}{c}39.02 \\
(0.46 \%)\end{array}$ \\
\hline 27-Feb-98 & Mar-98 & 250 & $\begin{array}{c}30.25 \\
(0.35 \%)\end{array}$ & $\begin{array}{c}45.57 \\
(0.53 \%)\end{array}$ \\
\hline
\end{tabular}

* Calculada mediante la estrategia teórica. 
En el cuadro 7, se presentan los resultados de determinar el valor teórico de la opción en cada subasta, para el caso hipotético en el que se varía la vigencia de la opción entre 1 y 20 días. En este cuadro se observa como en las primeras subastas los participantes valuaban la opción como si tuviese una vigencia de un día. A medida que transcurrió el programa, la vigencia implícita en el precio pagado por las opciones ha ido aumentando, lo que demuestra que gradualmente se está corrigiendo la subvaluación de la opción. 
Cuadro 7

Prima Estimada de Acuerdo a la Estrategia Teórica

(Porciento)

\begin{tabular}{|c|c|c|c|c|c|c|c|c|c|c|c|}
\hline \multirow{2}{*}{$\begin{array}{c}\text { Fecha de la } \\
\text { Subasta }\end{array}$} & \multirow[b]{2}{*}{ M-S* } & \multicolumn{10}{|c|}{ Días de Vigencia Restantes } \\
\hline & & 1 & 2 & 3 & 4 & 5 & 6 & 7 & 8 & 9 & 10 \\
\hline 7-Ago-96 & $0.510 \%$ & $0.255 \%$ & $0.333 \%$ & $0.376 \%$ & $0.420 \%$ & $0.453 \%$ & $0.473 \%$ & $0.504 \%$ & $0.525 \%$ & $0.545 \%$ & $0.562 \%$ \\
\hline 30-Ago-96 & $-0.437 \%$ & $0.000 \%$ & $0.080 \%$ & $0.143 \%$ & $0.193 \%$ & $0.236 \%$ & $0.272 \%$ & $0.304 \%$ & $0.333 \%$ & $0.359 \%$ & $0.383 \%$ \\
\hline 30-Sep-96 & $-0.054 \%$ & $0.000 \%$ & $0.135 \%$ & $0.215 \%$ & $0.273 \%$ & $0.319 \%$ & $0.357 \%$ & $0.390 \%$ & $0.418 \%$ & $0.444 \%$ & $0.467 \%$ \\
\hline $31-$ Oct-96 & $-1.603 \%$ & $0.000 \%$ & $0.041 \%$ & $0.101 \%$ & $0.161 \%$ & $0.216 \%$ & $0.267 \%$ & $0.313 \%$ & $0.355 \%$ & $0.394 \%$ & $0.430 \%$ \\
\hline 29-Nov-96 & $0.072 \%$ & $0.183 \%$ & $0.203 \%$ & $0.234 \%$ & $0.260 \%$ & $0.282 \%$ & $0.297 \%$ & $0.315 \%$ & $0.335 \%$ & $0.349 \%$ & $0.362 \%$ \\
\hline 30-Dic-96 & $0.100 \%$ & $0.206 \%$ & $0.313 \%$ & $0.366 \%$ & $0.403 \%$ & $0.431 \%$ & $0.454 \%$ & $0.472 \%$ & $0.487 \%$ & $0.504 \%$ & $0.515 \%$ \\
\hline 31-Ene-97 & $-0.008 \%$ & $0.000 \%$ & $0.120 \%$ & $0.190 \%$ & $0.240 \%$ & $0.279 \%$ & $0.312 \%$ & $0.340 \%$ & $0.365 \%$ & $0.386 \%$ & $0.406 \%$ \\
\hline 21-Feb-97 & $0.235 \%$ & $0.197 \%$ & $0.237 \%$ & $0.275 \%$ & $0.299 \%$ & $0.323 \%$ & $0.344 \%$ & $0.363 \%$ & $0.379 \%$ & $0.394 \%$ & $0.413 \%$ \\
\hline 28-Feb-97 & $-0.950 \%$ & $0.000 \%$ & $0.040 \%$ & $0.087 \%$ & $0.132 \%$ & $0.172 \%$ & $0.208 \%$ & $0.240 \%$ & $0.270 \%$ & $0.297 \%$ & $0.322 \%$ \\
\hline 31-Mar-97 & $0.150 \%$ & $0.163 \%$ & $0.192 \%$ & $0.220 \%$ & $0.244 \%$ & $0.260 \%$ & $0.278 \%$ & $0.297 \%$ & $0.311 \%$ & $0.319 \%$ & $0.334 \%$ \\
\hline 15-Abr-97 & $0.204 \%$ & $0.150 \%$ & $0.187 \%$ & $0.212 \%$ & $0.234 \%$ & $0.253 \%$ & $0.270 \%$ & $0.283 \%$ & $0.297 \%$ & $0.307 \%$ & $0.319 \%$ \\
\hline $30-A b r-97$ & $-0.249 \%$ & $0.000 \%$ & $0.061 \%$ & $0.106 \%$ & $0.142 \%$ & $0.171 \%$ & $0.197 \%$ & $0.218 \%$ & $0.238 \%$ & $0.255 \%$ & $0.271 \%$ \\
\hline 30-May-97 & $-0.083 \%$ & $0.000 \%$ & $0.087 \%$ & $0.142 \%$ & $0.182 \%$ & $0.214 \%$ & $0.241 \%$ & $0.264 \%$ & $0.284 \%$ & $0.303 \%$ & $0.319 \%$ \\
\hline 30-Jun-97 & $0.008 \%$ & $0.000 \%$ & $0.094 \%$ & $0.150 \%$ & $0.189 \%$ & $0.220 \%$ & $0.246 \%$ & $0.268 \%$ & $0.287 \%$ & $0.304 \%$ & $0.320 \%$ \\
\hline 9-Jul-97 & $0.254 \%$ & $0.152 \%$ & $0.193 \%$ & $0.220 \%$ & $0.243 \%$ & $0.262 \%$ & $0.279 \%$ & $0.292 \%$ & $0.301 \%$ & $0.317 \%$ & $0.323 \%$ \\
\hline 31-Jul-97 & $0.206 \%$ & $0.163 \%$ & $0.200 \%$ & $0.229 \%$ & $0.252 \%$ & $0.272 \%$ & $0.290 \%$ & $0.305 \%$ & $0.319 \%$ & $0.332 \%$ & $0.343 \%$ \\
\hline 29-Ago-97 & $-0.025 \%$ & $0.000 \%$ & $0.068 \%$ & $0.109 \%$ & $0.138 \%$ & $0.161 \%$ & $0.180 \%$ & $0.197 \%$ & $0.211 \%$ & $0.224 \%$ & $0.236 \%$ \\
\hline 30-Sep-97 & $-0.442 \%$ & $0.000 \%$ & $0.023 \%$ & $0.049 \%$ & $0.072 \%$ & $0.092 \%$ & $0.110 \%$ & $0.127 \%$ & $0.142 \%$ & $0.155 \%$ & $0.168 \%$ \\
\hline 31-Oct-97 & $-5.865 \%$ & $0.000 \%$ & $0.000 \%$ & $0.005 \%$ & $0.019 \%$ & $0.040 \%$ & $0.067 \%$ & $0.097 \%$ & $0.130 \%$ & $0.164 \%$ & $0.198 \%$ \\
\hline 28-Nov-97 & $0.684 \%$ & $0.331 \%$ & $0.440 \%$ & $0.506 \%$ & $0.548 \%$ & $0.589 \%$ & $0.632 \%$ & $0.655 \%$ & $0.681 \%$ & $0.716 \%$ & $0.727 \%$ \\
\hline 15-Dic-97 & $0.273 \%$ & $0.268 \%$ & $0.320 \%$ & $0.366 \%$ & $0.405 \%$ & $0.431 \%$ & $0.460 \%$ & $0.484 \%$ & $0.514 \%$ & $0.534 \%$ & $0.553 \%$ \\
\hline 30-Dic-97 & $0.333 \%$ & $0.202 \%$ & $0.250 \%$ & $0.286 \%$ & $0.310 \%$ & $0.334 \%$ & $0.354 \%$ & $0.372 \%$ & $0.387 \%$ & $0.402 \%$ & $0.414 \%$ \\
\hline 30-Ene-98 & $-1.162 \%$ & $0.000 \%$ & $0.024 \%$ & $0.062 \%$ & $0.101 \%$ & $0.137 \%$ & $0.171 \%$ & $0.202 \%$ & $0.231 \%$ & $0.257 \%$ & $0.282 \%$ \\
\hline 27-Feb-98 & $-0.146 \%$ & $0.000 \%$ & $0.105 \%$ & $0.171 \%$ & $0.221 \%$ & $0.261 \%$ & $0.295 \%$ & $0.324 \%$ & $0.349 \%$ & $0.372 \%$ & $0.393 \%$ \\
\hline
\end{tabular}

\begin{tabular}{|c|c|c|c|c|c|c|c|c|c|c|c|}
\hline \multirow{2}{*}{$\begin{array}{c}\text { Fecha de la } \\
\text { Subasta }\end{array}$} & \multicolumn{10}{|c|}{ Días de Vigencia Restantes } & \multirow{2}{*}{$\begin{array}{c}\text { Prima } \\
\text { Pond.** }\end{array}$} \\
\hline & 11 & 12 & 13 & 14 & 15 & 16 & 17 & 18 & 19 & 20 & \\
\hline 7-Ago-96 & $0.570 \%$ & $0.593 \%$ & $0.607 \%$ & $0.611 \%$ & $0.631 \%$ & $0.643 \%$ & $0.646 \%$ & $0.658 \%$ & $0.673 \%$ & $0.674 \%$ & $0.155 \%$ \\
\hline 30-Ago-96 & $0.404 \%$ & $0.425 \%$ & $0.443 \%$ & $0.460 \%$ & $0.477 \%$ & $0.492 \%$ & $0.507 \%$ & $0.520 \%$ & $0.533 \%$ & $0.546 \%$ & $0.061 \%$ \\
\hline 30-Sep-96 & $0.488 \%$ & $0.508 \%$ & $0.526 \%$ & $0.543 \%$ & $0.559 \%$ & $0.573 \%$ & $0.587 \%$ & $0.601 \%$ & $0.613 \%$ & $0.625 \%$ & $0.135 \%$ \\
\hline 31-Oct-96 & $0.464 \%$ & $0.496 \%$ & $0.525 \%$ & $0.553 \%$ & $0.579 \%$ & $0.604 \%$ & $0.628 \%$ & $0.650 \%$ & $0.671 \%$ & $0.692 \%$ & $0.128 \%$ \\
\hline 29-Nov-96 & $0.374 \%$ & $0.386 \%$ & $0.391 \%$ & $0.406 \%$ & $0.415 \%$ & $0.420 \%$ & $0.428 \%$ & $0.441 \%$ & $0.442 \%$ & $0.451 \%$ & $0.183 \%$ \\
\hline 30-Dic-96 & $0.529 \%$ & $0.534 \%$ & $0.549 \%$ & $0.561 \%$ & $0.563 \%$ & $0.576 \%$ & $0.587 \%$ & $0.594 \%$ & $0.595 \%$ & $0.608 \%$ & $0.175 \%$ \\
\hline 31-Ene-97 & $0.424 \%$ & $0.441 \%$ & $0.456 \%$ & $0.471 \%$ & $0.484 \%$ & $0.496 \%$ & $0.508 \%$ & $0.519 \%$ & $0.530 \%$ & $0.540 \%$ & $0.206 \%$ \\
\hline 21-Feb-97 & $0.421 \%$ & $0.437 \%$ & $0.443 \%$ & $0.453 \%$ & $0.461 \%$ & $0.470 \%$ & $0.480 \%$ & $0.486 \%$ & $0.494 \%$ & $0.503 \%$ & $0.194 \%$ \\
\hline 28-Feb-97 & $0.345 \%$ & $0.367 \%$ & $0.387 \%$ & $0.406 \%$ & $0.423 \%$ & $0.440 \%$ & $0.456 \%$ & $0.471 \%$ & $0.486 \%$ & $0.500 \%$ & $0.130 \%$ \\
\hline 31-Mar-97 & $0.340 \%$ & $0.355 \%$ & $0.365 \%$ & $0.368 \%$ & $0.381 \%$ & $0.389 \%$ & $0.391 \%$ & $0.402 \%$ & $0.409 \%$ & $0.410 \%$ & $0.276 \%$ \\
\hline 15-Abr-97 & $0.328 \%$ & $0.338 \%$ & $0.345 \%$ & $0.354 \%$ & $0.361 \%$ & $0.367 \%$ & $0.375 \%$ & $0.381 \%$ & $0.385 \%$ & $0.391 \%$ & $0.176 \%$ \\
\hline 30-Abr-97 & $0.286 \%$ & $0.300 \%$ & $0.312 \%$ & $0.324 \%$ & $0.335 \%$ & $0.345 \%$ & $0.355 \%$ & $0.364 \%$ & $0.373 \%$ & $0.381 \%$ & $0.181 \%$ \\
\hline 30-May-97 & $0.334 \%$ & $0.348 \%$ & $0.361 \%$ & $0.374 \%$ & $0.385 \%$ & $0.395 \%$ & $0.405 \%$ & $0.414 \%$ & $0.423 \%$ & $0.432 \%$ & $0.148 \%$ \\
\hline 30-Jun-97 & $0.334 \%$ & $0.348 \%$ & $0.360 \%$ & $0.371 \%$ & $0.382 \%$ & $0.392 \%$ & $0.401 \%$ & $0.410 \%$ & $0.418 \%$ & $0.427 \%$ & $0.259 \%$ \\
\hline 9-Jul-97 & $0.337 \%$ & $0.341 \%$ & $0.354 \%$ & $0.357 \%$ & $0.369 \%$ & $0.376 \%$ & $0.378 \%$ & $0.385 \%$ & $0.394 \%$ & $0.400 \%$ & $0.248 \%$ \\
\hline 31-Jul-97 & $0.353 \%$ & $0.363 \%$ & $0.372 \%$ & $0.381 \%$ & $0.387 \%$ & $0.396 \%$ & $0.403 \%$ & $0.404 \%$ & $0.415 \%$ & $0.422 \%$ & $0.299 \%$ \\
\hline 29-Ago-97 & $0.247 \%$ & $0.257 \%$ & $0.266 \%$ & $0.275 \%$ & $0.283 \%$ & $0.290 \%$ & $0.297 \%$ & $0.304 \%$ & $0.311 \%$ & $0.317 \%$ & $0.157 \%$ \\
\hline 30-Sep-97 & $0.179 \%$ & $0.190 \%$ & $0.200 \%$ & $0.210 \%$ & $0.219 \%$ & $0.227 \%$ & $0.235 \%$ & $0.243 \%$ & $0.250 \%$ & $0.257 \%$ & $0.210 \%$ \\
\hline 31-Oct-97 & $0.232 \%$ & $0.266 \%$ & $0.300 \%$ & $0.333 \%$ & $0.365 \%$ & $0.397 \%$ & $0.428 \%$ & $0.458 \%$ & $0.487 \%$ & $0.515 \%$ & $0.160 \%$ \\
\hline 28-Nov-97 & $0.757 \%$ & $0.766 \%$ & $0.793 \%$ & $0.799 \%$ & $0.813 \%$ & $0.838 \%$ & $0.841 \%$ & $0.853 \%$ & $0.875 \%$ & $0.887 \%$ & $0.370 \%$ \\
\hline 15-Dic-97 & $0.570 \%$ & $0.586 \%$ & $0.594 \%$ & $0.614 \%$ & $0.618 \%$ & $0.631 \%$ & $0.650 \%$ & $0.652 \%$ & $0.663 \%$ & $0.675 \%$ & $0.415 \%$ \\
\hline 30-Dic-97 & $0.427 \%$ & $0.432 \%$ & $0.448 \%$ & $0.458 \%$ & $0.460 \%$ & $0.475 \%$ & $0.483 \%$ & $0.484 \%$ & $0.493 \%$ & $0.500 \%$ & $0.416 \%$ \\
\hline 30-Ene-98 & $0.305 \%$ & $0.326 \%$ & $0.347 \%$ & $0.366 \%$ & $0.383 \%$ & $0.401 \%$ & $0.417 \%$ & $0.432 \%$ & $0.447 \%$ & $0.461 \%$ & $0.187 \%$ \\
\hline 27-Feb-98 & $0.412 \%$ & $0.430 \%$ & $0.446 \%$ & $0.461 \%$ & $0.475 \%$ & $0.488 \%$ & $0.501 \%$ & $0.512 \%$ & $0.524 \%$ & $0.535 \%$ & $0.355 \%$ \\
\hline
\end{tabular}




\section{Cuadro 7 (continuación)}

(Pesos por Mil USD)

\begin{tabular}{|c|c|c|c|c|c|c|c|c|c|c|c|}
\hline \multirow{2}{*}{$\begin{array}{c}\text { Fecha de la } \\
\text { Subasta }\end{array}$} & \multirow[b]{2}{*}{ M-S* } & \multicolumn{10}{|c|}{ Días de Vigencia Restantes } \\
\hline & & 1 & 2 & 3 & 4 & 5 & 6 & 7 & 8 & 9 & 10 \\
\hline 7-Ago-96 & $0.510 \%$ & 19.14 & 25.00 & 28.25 & 31.57 & 34.00 & 35.53 & 37.87 & 39.43 & 40.91 & 42.22 \\
\hline 30-Ago-96 & $-0.437 \%$ & 0.00 & 6.07 & 10.82 & 14.66 & 17.87 & 20.65 & 23.09 & 25.26 & 27.21 & 29.02 \\
\hline 30-Sep-96 & $-0.054 \%$ & 0.00 & 10.18 & 16.21 & 20.63 & 24.09 & 26.97 & 29.42 & 31.59 & 33.53 & 35.27 \\
\hline 31-Oct-96 & $-1.603 \%$ & 0.00 & 3.32 & 8.15 & 12.94 & 17.39 & 21.46 & 25.18 & 28.58 & 31.72 & 34.63 \\
\hline 29-Nov-96 & $0.072 \%$ & 14.46 & 16.05 & 18.44 & 20.51 & 22.29 & 23.47 & 24.83 & 26.45 & 27.56 & 28.61 \\
\hline 30-Dic-96 & $0.100 \%$ & 16.21 & 24.57 & 28.77 & 31.67 & 33.86 & 35.63 & 37.11 & 38.28 & 39.60 & 40.44 \\
\hline 31-Ene-97 & $-0.008 \%$ & 0.00 & 9.38 & 14.84 & 18.79 & 21.85 & 24.42 & 26.61 & 28.52 & 30.21 & 31.75 \\
\hline 21-Feb-97 & $0.235 \%$ & 15.30 & 18.38 & 21.34 & 23.24 & 25.11 & 26.73 & 28.17 & 29.41 & 30.62 & 32.06 \\
\hline 28-Feb-97 & $-0.950 \%$ & 0.00 & 3.15 & 6.95 & 10.51 & 13.72 & 16.59 & 19.17 & 21.52 & 23.67 & 25.67 \\
\hline 31-Mar-97 & $0.150 \%$ & 12.94 & 15.24 & 17.45 & 19.34 & 20.64 & 22.01 & 23.58 & 24.66 & 25.27 & 26.49 \\
\hline 15-Abr-97 & $0.204 \%$ & 11.85 & 14.78 & 16.70 & 18.48 & 19.97 & 21.27 & 22.36 & 23.39 & 24.24 & 25.14 \\
\hline $30-A b r-97$ & $-0.249 \%$ & 0.00 & 4.88 & 8.45 & 11.29 & 13.63 & 15.63 & 17.37 & 18.90 & 20.30 & 21.57 \\
\hline 30-May-97 & $-0.083 \%$ & 0.00 & 6.92 & 11.20 & 14.41 & 16.96 & 19.09 & 20.90 & 22.51 & 23.96 & 25.26 \\
\hline 30-Jun-97 & $0.008 \%$ & 0.00 & 7.50 & 11.91 & 15.00 & 17.46 & 19.54 & 21.29 & 22.81 & 24.19 & 25.45 \\
\hline 9-Jul-97 & $0.254 \%$ & 11.99 & 15.20 & 17.41 & 19.19 & 20.70 & 22.00 & 23.07 & 23.76 & 25.04 & 25.51 \\
\hline 31-Jul-97 & $0.206 \%$ & 12.75 & 15.65 & 17.89 & 19.74 & 21.32 & 22.72 & 23.87 & 24.99 & 25.97 & 26.86 \\
\hline 29-Ago-97 & $-0.025 \%$ & 0.00 & 5.27 & 8.46 & 10.74 & 12.55 & 14.04 & 15.32 & 16.45 & 17.46 & 18.36 \\
\hline 30-Sep-97 & $-0.442 \%$ & 0.00 & 1.80 & 3.79 & 5.59 & 7.19 & 8.63 & 9.91 & 11.06 & 12.13 & 13.11 \\
\hline 31-Oct-97 & $-5.865 \%$ & 0.00 & 0.01 & 0.42 & 1.55 & 3.33 & 5.59 & 8.14 & 10.87 & 13.70 & 16.57 \\
\hline 28-Nov-97 & $0.684 \%$ & 27.15 & 36.11 & 41.56 & 44.99 & 48.38 & 51.91 & 53.76 & 55.97 & 58.77 & 59.71 \\
\hline 15-Dic-97 & $0.273 \%$ & 21.81 & 26.07 & 29.77 & 32.99 & 35.11 & 37.42 & 39.42 & 41.83 & 43.44 & 45.00 \\
\hline 30-Dic-97 & $0.333 \%$ & 16.28 & 20.20 & 23.02 & 24.98 & 26.91 & 28.55 & 30.04 & 31.20 & 32.43 & 33.35 \\
\hline 30-Ene-98 & $-1.162 \%$ & 0.00 & 2.00 & 5.23 & 8.54 & 11.63 & 14.49 & 17.11 & 19.54 & 21.78 & 23.87 \\
\hline 27-Feb-98 & $-0.146 \%$ & 0.00 & 8.92 & 14.57 & 18.85 & 22.28 & 25.13 & 27.58 & 29.75 & 31.74 & 33.50 \\
\hline
\end{tabular}

\begin{tabular}{|c|c|c|c|c|c|c|c|c|c|c|c|}
\hline \multirow{2}{*}{$\begin{array}{c}\text { Fecha de la } \\
\text { Subasta }\end{array}$} & \multicolumn{10}{|c|}{ Días de Vigencia Restantes } & \multirow{2}{*}{$\begin{array}{l}\text { Prima } \\
\text { Pond.** }\end{array}$} \\
\hline & 11 & 12 & 13 & 14 & 15 & 16 & 17 & 18 & 19 & 20 & \\
\hline 7-Ago-96 & 42.83 & 44.52 & 45.58 & 45.93 & 47.43 & 48.29 & 48.50 & 49.41 & 50.56 & 50.67 & 11.68 \\
\hline 30-Ago-96 & 30.66 & 32.20 & 33.59 & 34.92 & 36.16 & 37.32 & 38.42 & 39.45 & 40.44 & 41.39 & 4.65 \\
\hline 30-Sep-96 & 36.87 & 38.36 & 39.72 & 41.03 & 42.18 & 43.30 & 44.36 & 45.36 & 46.30 & 47.19 & 10.18 \\
\hline 31-Oct-96 & 37.35 & 39.89 & 42.28 & 44.51 & 46.61 & 48.62 & 50.51 & 52.31 & 54.03 & 55.68 & 10.32 \\
\hline 29-Nov-96 & 29.55 & 30.46 & 30.83 & 32.05 & 32.79 & 33.14 & 33.80 & 34.77 & 34.89 & 35.57 & 14.43 \\
\hline 30-Dic-96 & 41.59 & 41.95 & 43.11 & 44.08 & 44.26 & 45.25 & 46.10 & 46.70 & 46.74 & 47.74 & 13.75 \\
\hline 31-Ene-97 & 33.17 & 34.49 & 35.68 & 36.82 & 37.85 & 38.82 & 39.75 & 40.63 & 41.47 & 42.24 & 16.14 \\
\hline 21-Feb-97 & 32.66 & 33.93 & 34.39 & 35.20 & 35.80 & 36.49 & 37.24 & 37.74 & 38.34 & 39.02 & 15.08 \\
\hline 28-Feb-97 & 27.51 & 29.24 & 30.85 & 32.35 & 33.77 & 35.11 & 36.37 & 37.58 & 38.73 & 39.83 & 10.40 \\
\hline 31-Mar-97 & 27.00 & 28.18 & 28.92 & 29.19 & 30.22 & 30.83 & 31.00 & 31.92 & 32.46 & 32.54 & 21.86 \\
\hline 15-Abr-97 & 25.84 & 26.63 & 27.19 & 27.90 & 28.48 & 28.95 & 29.57 & 30.06 & 30.39 & 30.85 & 13.86 \\
\hline 30-Abr-97 & 22.75 & 23.82 & 24.82 & 25.77 & 26.63 & 27.46 & 28.24 & 28.96 & 29.66 & 30.33 & 14.38 \\
\hline 30-May-97 & 26.46 & 27.57 & 28.60 & 29.58 & 30.44 & 31.27 & 32.06 & 32.81 & 33.51 & 34.17 & 11.74 \\
\hline 30-Jun-97 & 26.58 & 27.63 & 28.61 & 29.51 & 30.36 & 31.16 & 31.90 & 32.60 & 33.27 & 33.92 & 20.61 \\
\hline 9-Jul-97 & 26.63 & 26.95 & 27.99 & 28.22 & 29.15 & 29.71 & 29.84 & 30.41 & 31.14 & 31.60 & 19.57 \\
\hline 31-Jul-97 & 27.60 & 28.43 & 29.13 & 29.78 & 30.27 & 30.96 & 31.51 & 31.61 & 32.49 & 32.99 & 23.36 \\
\hline 29-Ago-97 & 19.21 & 19.99 & 20.72 & 21.39 & 22.01 & 22.60 & 23.16 & 23.67 & 24.18 & 24.65 & 12.27 \\
\hline 30-Sep-97 & 14.02 & 14.86 & 15.65 & 16.39 & 17.09 & 17.74 & 18.37 & 18.97 & 19.53 & 20.07 & 16.10 \\
\hline 31-Oct-97 & 19.44 & 22.30 & 25.11 & 27.87 & 30.58 & 33.22 & 35.80 & 38.31 & 40.75 & 43.13 & 13.05 \\
\hline 28-Nov-97 & 62.20 & 62.89 & 65.11 & 65.61 & 66.81 & 68.81 & 69.09 & 70.10 & 71.87 & 72.88 & 30.05 \\
\hline 15-Dic-97 & 46.37 & 47.68 & 48.35 & 49.96 & 50.33 & 51.32 & 52.90 & 53.10 & 53.93 & 54.93 & 33.81 \\
\hline 30-Dic-97 & 34.42 & 34.83 & 36.11 & 36.91 & 37.13 & 38.28 & 38.93 & 39.05 & 39.77 & 40.31 & 33.54 \\
\hline 30-Ene-98 & 25.81 & 27.64 & 29.34 & 30.95 & 32.46 & 33.90 & 35.28 & 36.58 & 37.82 & 39.02 & 15.85 \\
\hline 27-Feb-98 & 35.13 & 36.62 & 38.00 & 39.29 & 40.46 & 41.61 & 42.69 & 43.68 & 44.65 & 45.57 & 30.25 \\
\hline
\end{tabular}

* M-S representa la diferencia porcentual entre la media móvil del tipo de cambio y el nivel de este en la fecha de la subasta.

** Es la prima ponderada observada en la subasta de la opción.

Nota: Los datos sombreados son aquellos que más se asemejan a la prima observada. 
Existen por lo menos dos razones adicionales para explicar la subvaluación del precio de las opciones. Primero, debido al mecanismo de subastas utilizado, el precio, puede no reflejar fielmente la valuación hecha por los participantes en dicha subasta. En segundo lugar, otra razón para la subvaluación del precio de las opciones es que el supuesto que se hizo sobre la distribución de las fluctuaciones cambiarias en el modelo teórico puede no ser el correcto. Podemos suponer, como distribución alternativa, que las fluctuaciones cambiarias se distribuyen normalmente con una probabilidad (1-P) y con probabilidad $\mathrm{P}$, el tipo de cambio se deprecia en $5 \%$ en un día. Haciendo este supuesto y fijando $\mathrm{P}=0.5 \%$, podemos calcular cual es la media y varianza de la distribución normal que hacen que, la tendencia y varianza de las fluctuaciones cambiarias sean iguales a las implícitas en las coberturas y en las opciones sobre los futuros. Con base en estos supuestos se recalcula un nuevo precio teórico de la opción que se compara con el anterior y con el observado. Estos resultados se presentan en el cuadro 8. Este ejercicio indica que en algunos casos esta hipótesis es capaz de explicar una fracción importante de la subvaluación del precio de la opción, como por ejemplo para los casos de las subastas de marzo, junio, julio, agosto y septiembre de $1997^{13}$.

\footnotetext{
${ }^{13}$ En este caso la probabilidad de ejercicio variará inversamente con la probabilidad de una devaluación discreta $(P)$.
} 


\section{Cuadro 8}

Prima Pagada por la Opción

(\$ x Mil Dls)

\begin{tabular}{|c|c|c|c|}
\hline \multirow{2}{*}{$\begin{array}{c}\text { Fecha } \\
\text { de la } \\
\text { Subasta }\end{array}$} & \multirow[b]{2}{*}{$\begin{array}{c}\text { Observada } \\
\text { Subasta }\end{array}$} & \multicolumn{2}{|c|}{ Estrategia Teórica } \\
\hline & & $\begin{array}{c}\text { Probabilidad } \\
\text { Normal }\end{array}$ & $\begin{array}{l}\text { Probabilidad } \\
\text { Devaluación* }\end{array}$ \\
\hline \multirow[t]{2}{*}{ 7-Ago-96 } & 11.68 & 51.31 & 48.83 \\
\hline & $(0.16 \%)$ & $(0.68 \%)$ & $(0.65 \%)$ \\
\hline \multirow[t]{2}{*}{ 30-Ago-96 } & 4.65 & 40.36 & 40.19 \\
\hline & $(0.06 \%)$ & $(0.53 \%)$ & $(0.53 \%)$ \\
\hline \multirow[t]{2}{*}{ 30-Sep-96 } & 10.18 & 52.06 & 44.68 \\
\hline & $(0.13 \%)$ & $(0.69 \%)$ & $(0.59 \%)$ \\
\hline \multirow[t]{2}{*}{ 31-Oct-96 } & 10.32 & 46.68 & 30.58 \\
\hline & $(0.13 \%)$ & $(0.58 \%)$ & $(0.38 \%)$ \\
\hline \multirow[t]{2}{*}{ 29-Nov-96 } & 14.43 & 36.54 & 30.23 \\
\hline & $(0.18 \%)$ & $(0.46 \%)$ & $(0.38 \%)$ \\
\hline \multirow[t]{2}{*}{ 30-Dic-96 } & 13.75 & 32.33 & 27.16 \\
\hline & $(0.18 \%)$ & $(0.41 \%)$ & $(0.35 \%)$ \\
\hline \multirow[t]{2}{*}{ 31-Ene-96 } & 16.14 & 41.94 & 37.11 \\
\hline & $(0.21 \%)$ & $(0.57 \%)$ & $(0.50 \%)$ \\
\hline \multirow[t]{2}{*}{ 21-Feb-97 } & 15.08 & 30.15 & 25.12 \\
\hline & $(0.19 \%)$ & $(0.39 \%)$ & $(0.32 \%)$ \\
\hline \multirow[t]{2}{*}{ 28-Feb-97 } & 10.40 & 19.95 & 16.67 \\
\hline & $(0.13 \%)$ & $(0.25 \%)$ & $(0.21 \%)$ \\
\hline \multirow[t]{2}{*}{ 31-Mar-97 } & 21.86 & 37.09 & 25.28 \\
\hline & $(0.28 \%)$ & $(0.47 \%)$ & $(0.32 \%)$ \\
\hline \multirow[t]{2}{*}{ 15-Abr-97 } & 13.86 & 26.76 & 18.85 \\
\hline & $(0.18 \%)$ & $(0.34 \%)$ & $(0.24 \%)$ \\
\hline \multirow[t]{2}{*}{ 30-Abr-97 } & 14.38 & 32.51 & 24.29 \\
\hline & $(0.18 \%)$ & $(0.41 \%)$ & $(0.31 \%)$ \\
\hline \multirow[t]{2}{*}{ 30-May-97 } & 11.74 & 32.65 & 29.17 \\
\hline & $(0.15 \%)$ & $(0.41 \%)$ & $(0.37 \%)$ \\
\hline \multirow[t]{2}{*}{ 30-Jun-97 } & 20.61 & 39.23 & 25.35 \\
\hline & $(0.26 \%)$ & $(0.49 \%)$ & $(0.32 \%)$ \\
\hline \multirow[t]{2}{*}{ 9-Jul-97 } & 19.57 & 32.99 & 22.12 \\
\hline & $(0.25 \%)$ & $(0.42 \%)$ & $(0.28 \%)$ \\
\hline \multirow[t]{2}{*}{ 31-Jul-97 } & 23.36 & 37.55 & 24.10 \\
\hline & $(0.30 \%)$ & $(0.48 \%)$ & $(0.31 \%)$ \\
\hline \multirow[t]{2}{*}{ 29-Ago-97 } & 12.27 & 24.13 & 14.48 \\
\hline & $(0.16 \%)$ & $(0.31 \%)$ & $(0.19 \%)$ \\
\hline \multirow[t]{2}{*}{ 30-Sep-97 } & 16.10 & 20.07 & 14.24 \\
\hline & $(0.21 \%)$ & $(0.26 \%)$ & $(0.18 \%)$ \\
\hline \multirow[t]{2}{*}{$31-$ Oct-97 } & 13.05 & 43.13 & 39.12 \\
\hline & $(0.16 \%)$ & $(0.52 \%)$ & $(0.47 \%)$ \\
\hline 28-Nov-97 & 30.05 & 72.88 & 66.19 \\
\hline & $(0.37 \%)$ & $(0.89 \%)$ & $(0.81 \%)$ \\
\hline 15-Dic-97 & 33.81 & 54.93 & 51.27 \\
\hline & $(0.42 \%)$ & $(0.68 \%)$ & $(0.63 \%)$ \\
\hline 30-Dic-97 & 33.54 & 40.31 & 42.29 \\
\hline & $(0.42 \%)$ & $(0.50 \%)$ & $(0.52 \%)$ \\
\hline 30-Ene-98 & 15.85 & 39.02 & 37.41 \\
\hline & $(0.19 \%)$ & $(0.46 \%)$ & $(0.44 \%)$ \\
\hline 27-Feb-98 & 30.25 & 45.57 & 42.44 \\
\hline & $(0.35 \%)$ & $(0.53 \%)$ & $(0.50 \%)$ \\
\hline
\end{tabular}

* Se asume que con probabilidad de $0.5 \%$ se presenta una devaluación de $5 \%$ en el tipo de cambio. 
Por último, en el cuadro 9, comparamos las decisiones de ejercicio observadas en el mercado con las decisiones de ejercicio derivadas según la metodología de la sección III. En esta comparación, se hace evidente que estas opciones han sido ejercidas cuando se observaron apreciaciones menores a las indicadas por la función de ejercicio óptimo. Esta evidencia le resta validez a la explicación de la subvaluación debida a imperfecciones de la subasta, ya que una vez que un individuo compra la opción, independientemente del precio pagado por ella, debe comportarse de acuerdo al supuesto de maximización de ganancias implícito en la metodología utilizada en este trabajo. Sin embargo, esta observación

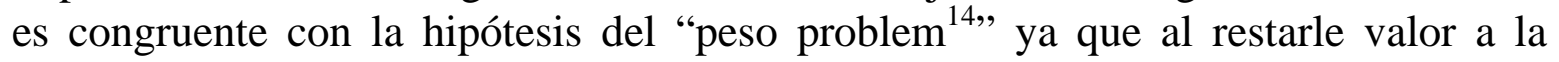
opción los dueños de la opción son menos exigentes y la ejercerán antes de lo indicado por la simulación que asume una distribución normal de las fluctuaciones cambiarias. Cabe destacar que de haber seguido la estrategia óptima, que el modelo y los parámetros utilizados sugiere, las opciones se hubiesen ejercido en su totalidad en 20 de las 24 subastas consideradas y la ganancia promedio hubiese sido de $0.59 \%$, la cual se compara favorablemente con la ganancia promedio obtenida en la realidad, que fue de $0.20 \%$.

\footnotetext{
${ }^{14}$ Se refiere a la hipótesis presentada en la página anterior que en cada período exista una probabilidad muy pequeña de que el tipo de cambio experimente una depreciación brusca.
} 


\section{Cuadro 9}

\begin{tabular}{|c|c|c|c|c|c|c|c|c|c|c|}
\hline \multirow{2}{*}{$\begin{array}{c}\text { Fecha } \\
\text { de la } \\
\text { Subasta }\end{array}$} & & Fecha & Monto & Apreciación & & Gananc & oor Subasta & & Estrate & a Teórica \\
\hline & $\begin{array}{c}\text { de la } \\
\text { Opción }\end{array}$ & $\begin{array}{c}\text { de } \\
\text { Ejercicio }\end{array}$ & $\begin{array}{c}\text { Ejercido } \\
\text { (MDD) }\end{array}$ & $\begin{array}{c}\text { del T.C. } \\
\text { (Ganancia) }\end{array}$ & Máxima & Mínima & $\begin{array}{l}\text { Promedio } \\
\text { Ejercidas }\end{array}$ & $\begin{array}{c}\text { Promedio } \\
\text { Total }\end{array}$ & $\begin{array}{c}\text { Fecha } \\
\text { Ejercicio }\end{array}$ & $\begin{array}{c}\text { Apreciación } \\
\text { Observada }\end{array}$ \\
\hline 7-Ago-96 & Ago-96 & 9-Ago-96 & $\frac{105}{105}$ & $0.23 \%$ & $0.28 \%$ & $0.09 \%$ & $0.23 \%$ & $0.23 \%$ & 28-Ago-97 & $0.31 \%$ \\
\hline & & 12-Ago-96 & 10 & $0.09 \%$ & & & & & & \\
\hline & & 13-Ago-96 & 15 & $0.28 \%$ & & & & & & \\
\hline & & Subtotal & 130 & & & & & & & \\
\hline 30-Ago-96 & Sep-96 & 17-Sep-96 & 140 & $0.11 \%$ & $0.20 \%$ & $0.06 \%$ & $0.10 \%$ & $0.10 \%$ & 24-Sep-96 & $0.61 \%$ \\
\hline & & 18-Sep-96 & 15 & $0.20 \%$ & & & & & & \\
\hline & & 25-Sep-96 & 25 & $0.06 \%$ & & & & & & \\
\hline & & 27-Sep-96 & 20 & $0.00 \%$ & & & & & & \\
\hline & & Subtotal & 200 & & & & & & & \\
\hline 30-Sep-96 & Oct-96 & 2-Oct-96 & 179 & $0.18 \%$ & $0.18 \%$ & $0.18 \%$ & $0.18 \%$ & $0.16 \%$ & 28-Oct-96 & $0.81 \%$ \\
\hline & & Subtotal & 179 & & & & & & & \\
\hline $31-0 c t-96$ & Nov-96 & 19-Nov-96 & 77 & $0.14 \%$ & $0.44 \%$ & $0.02 \%$ & $0.23 \%$ & $0.23 \%$ & N.E. & \\
\hline & & 21-Nov-96 & 20 & $0.02 \%$ & & & & & & \\
\hline & & 22-Nov-96 & 93 & $0.33 \%$ & & & & & & \\
\hline & & 26-Nov-96 & 10 & $0.44 \%$ & & & & & & \\
\hline & & Subtotal & 200 & & & & & & & \\
\hline 29-Nov-96 & Dic-96 & 3-Dic-96 & 110 & $0.18 \%$ & $0.47 \%$ & $0.00 \%$ & $0.27 \%$ & $0.27 \%$ & 10-Dic-96 & $0.39 \%$ \\
\hline & & 17-Dic-96 & 15 & $0.00 \%$ & & & & & & \\
\hline & & 18-Dic-96 & 75 & $0.47 \%$ & & & & & & \\
\hline & & Subtotal & 200 & & & & & & & \\
\hline 30-Dic-96 & Ene-97 & 6-Ene-97 & 118 & $0.20 \%$ & $0.26 \%$ & $0.20 \%$ & $0.23 \%$ & $0.23 \%$ & 13-Ene-97 & $0.61 \%$ \\
\hline & & 8-Ene-97 & 62 & $0.26 \%$ & & & & & & \\
\hline & & 20-Ene-97 & 120 & & & & & & & \\
\hline & & Subtotal & 300 & & & & & & & \\
\hline 31-Ene-96 & Feb-97 & $11-$ Feb-97 & 138 & $0.08 \%$ & $0.15 \%$ & $0.08 \%$ & $0.11 \%$ & $0.11 \%$ & $20-F e b-97$ & $0.55 \%$ \\
\hline & & 13-Feb-97 & 50 & $0.09 \%$ & & & & & & \\
\hline & & 14-Feb-97 & 112 & $0.15 \%$ & & & & & & \\
\hline & & Subtotal & 300 & & & & & & & \\
\hline 21-Feb-97 & Feb-97 & $24-F e b-97$ & 148 & $0.15 \%$ & $0.15 \%$ & $0.15 \%$ & $0.15 \%$ & $0.07 \%$ & N.E. & \\
\hline & (segunda) & Subtotal & 148 & & & & & & & \\
\hline 28-Feb-97 & Mar-97 & 31-Mar-97 & 120 & $0.00 \%$ & $0.00 \%$ & $0.00 \%$ & $0.00 \%$ & $0.00 \%$ & 24-Mar-97 & $0.57 \%$ \\
\hline & & Subtotal & 120 & & & & & & & \\
\hline 31-Mar-97 & Abr-97 & 1-Abr-97 & 100 & $0.25 \%$ & $0.27 \%$ & $0.24 \%$ & $0.24 \%$ & $0.24 \%$ & 7-Abr-97 & $0.67 \%$ \\
\hline & & 8-Abr-97 & 50 & $0.27 \%$ & & & & & & \\
\hline & & 15-Abr-97 & 150 & $0.24 \%$ & & & & & & \\
\hline & & Subtotal & 300 & & & & & & & \\
\hline 15-Abr-97 & Abr-97 & 17-Abr-97 & 95 & $0.16 \%$ & $0.30 \%$ & $0.09 \%$ & $0.14 \%$ & $0.13 \%$ & 24-Abr-97 & $0.30 \%$ \\
\hline & (segunda) & 18-Abr-97 & 113 & $0.14 \%$ & & & & & & \\
\hline & & 22-Abr-97 & 47 & $0.09 \%$ & & & & & & \\
\hline & & $24-A b r-97^{*}$ & 8 & $0.30 \%$ & & & & & & \\
\hline & & Subtotal & 263 & & & & & & & \\
\hline 30-Abr-97 & May-97 & 19-Мay-97 & 100 & $0.01 \%$ & $0.28 \%$ & $0.01 \%$ & $0.10 \%$ & $0.10 \%$ & 6-May-97 & $0.42 \%$ \\
\hline & & 20-May-97 & 134 & $0.07 \%$ & & & & & & \\
\hline & & 21-May-97 & 66 & $0.28 \%$ & & & & & & \\
\hline & & Subtotal & 300 & & & & & & & \\
\hline 30-May-97 & Jun-97 & 25-Jun-97 & 20 & $0.00 \%$ & $0.00 \%$ & $0.00 \%$ & $0.00 \%$ & $0.00 \%$ & 18-Jun-97 & $0.41 \%$ \\
\hline & & Subtotal & 20 & & & & & & & \\
\hline 30-Jun-97 & Jul-97 & 1-Jul-97 & 218 & $0.26 \%$ & $0.49 \%$ & $0.25 \%$ & $0.27 \%$ & $0.27 \%$ & 7-Jul-97 & $0.74 \%$ \\
\hline & & 9-Jul-97 & 62 & $0.25 \%$ & & & & & & \\
\hline & & 11-Jul-97 & 20 & $0.49 \%$ & & & & & & \\
\hline & & Subtotal & 300 & & & & & & & \\
\hline 9-Jul-97 & Jul-97 & 10-Jul-97 & 279 & $0.05 \%$ & $0.49 \%$ & $0.05 \%$ & $0.08 \%$ & $0.08 \%$ & 11-Jul-97 & $0.49 \%$ \\
\hline & (segunda) & 11-Jul-97* & 21 & $0.49 \%$ & & & & & & \\
\hline & & Subtotal & 300 & & & & & & & \\
\hline 31-Jul-97 & Ago-97 & 5-Ago-97 & 225 & $0.33 \%$ & $0.33 \%$ & $0.11 \%$ & $0.29 \%$ & $0.29 \%$ & 25-Ago-97 & $0.41 \%$ \\
\hline & & 6-Ago-97 & 133 & $0.31 \%$ & & & & & & \\
\hline & & 19-Ago-97 & 102 & $0.23 \%$ & & & & & & \\
\hline & & 27-Ago-97 & 40 & $0.11 \%$ & & & & & & \\
\hline & & Subtotal & 500 & & & & & & & \\
\hline 29-Ago-97 & Sep-97 & 3-Sep-97 & 41 & $0.11 \%$ & $0.40 \%$ & $0.00 \%$ & $0.20 \%$ & $0.20 \%$ & 23-Sep-97 & $0.40 \%$ \\
\hline & & 17-Sep-97 & 235 & $0.17 \%$ & & & & & & \\
\hline & & 19-Sep-97 & 80 & $0.00 \%$ & & & & & & \\
\hline & & 22-Sep-97 & 144 & $0.40 \%$ & & & & & & \\
\hline & & Subtotal & 500 & & & & & & & \\
\hline 30-Sep-97 & Oct-97 & $1-$ Oct-97 & 167 & $0.80 \%$ & $0.80 \%$ & $0.00 \%$ & $0.54 \%$ & $0.51 \%$ & 1-Oct-97 & $0.80 \%$ \\
\hline & & 7-Oct-97 & 20 & $0.00 \%$ & & & & & & \\
\hline & & 20-Oct-97 & 188 & $0.37 \%$ & & & & & & \\
\hline & & Subtotal & 375 & & & & & & & \\
\hline 31-Oct-97 & Nov-97 & $25-N o v-97$ & 77 & $0.00 \%$ & $0.43 \%$ & $0.00 \%$ & $0.18 \%$ & $0.18 \%$ & 21-Nov-97 & $1.34 \%$ \\
\hline & & 26-Nov-97 & 98 & $0.43 \%$ & & & & & & \\
\hline & & $\begin{array}{l}\text { 28-Nov-97 } \\
\text { Subtotal }\end{array}$ & $\begin{array}{c}75 \\
250\end{array}$ & $0.04 \%$ & & & & & & \\
\hline 28-Nov-97 & Dic-97 & 1-Dic-97 & 121 & $0.40 \%$ & $0.76 \%$ & $0.33 \%$ & $0.46 \%$ & $0.46 \%$ & 15-Dic-97 & $0.76 \%$ \\
\hline & & 2-Dic-97 & 71 & $0.33 \%$ & & & & & & \\
\hline & & 15-Dic-97 & 58 & $0.76 \%$ & & & & & & \\
\hline 15-Dic-97 & Dic-97 & $\begin{array}{l}\text { Subtotal } \\
16 \text {-Dic-97 }\end{array}$ & $\frac{250}{195}$ & $0.57 \%$ & $0.57 \%$ & $0.24 \%$ & $0.49 \%$ & $0.49 \%$ & 29-Dic-97 & $0.73 \%$ \\
\hline & & 30-Dic-97 & 55 & $0.24 \%$ & & & & & & \\
\hline & & Subtotal & 250 & & & & & & & \\
\hline 30-Dic-97 & Ene-98 & 5-Ene-98 & 49 & $0.20 \%$ & $0.20 \%$ & $0.20 \%$ & $0.20 \%$ & $0.04 \%$ & N.E. & \\
\hline & & Subtotal & 49 & & & & & & & \\
\hline 30-Ene-98 & Feb-98 & No Ejercidas & 0 & $0.00 \%$ & $0.00 \%$ & $0.00 \%$ & $0.00 \%$ & $0.00 \%$ & N.E. & \\
\hline & & Subtotal & 0 & & & & & & & \\
\hline 27-Feb-98 & Mar-98 & 23-Mar-98 & 250 & $0.33 \%$ & $0.33 \%$ & $0.33 \%$ & $0.33 \%$ & $0.33 \%$ & 18-Mar-98 & $0.47 \%$ \\
\hline ercicin co & 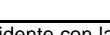 & Subtotal & 250 & & & & & & & \\
\hline
\end{tabular}


Cabe señalar que en ocho ocasiones no se ejerció la totalidad de los contratos vigentes y en aquellas fechas en que se presentó ejercicio de las opciones, este se hizo con una apreciación promedio de $0.21 \%$. Para las distintas fechas en las que se ejercieron opciones correspondientes a un mismo contrato, se calcularon las apreciaciones o ganancias máximas y mínimas correspondientes, observándose que la diferencia promedio entre éstas fue de 3.77 veces.

$\mathrm{Al}$ estimar la ganancia promedio total, ésta resultó de $0.20 \%$, la cual al ser comparada con el promedio de las primas pagadas por los tenedores de la opción en cada contrato, resulta menor por un monto aproximado de $0.02 \%$. Sin embargo, cabe señalar que en diez de los veinticuatro contratos considerados, la ganancia promedio excedió a la prima promedio. Además, en este estudio no se han considerado las fluctuaciones cambiarias observadas durante el día, éstas tenderán a aumentar el valor de la opción y la ganancia observada.

Al comparar las observaciones reales del comportamiento de los tenedores de las opciones con la estrategia teórica desarrollada en este trabajo, se observan los siguientes resultados:

i) La prima promedio pagada observada fue de 17.37 pesos por mil dólares, mientras que bajo la estrategia propuesta, habría sido de 38.74 pesos. En el caso hipotético en el cual la opción tiene una vigencia de un día la prima teórica promedio fue de 7.50 pesos.

ii) Debido a que la estrategia de ejercicio plantea que lo óptimo es ejercer la totalidad de las opciones en un solo momento en el que se presente una apreciación considerable, bajo la estrategia teórica se habría presentado sólo una fecha de ejercicio por la totalidad del monto subastado durante el mes de vigencia del contrato. Asimismo, según dicha estrategia teórica, en cuatro de los veinticuatro contratos considerados, no se hubiesen ejercido las opciones de venta, ya que ninguna apreciación observada lo justificó en base a la magnitud de esta y a la madurez de la opción.

iii) Parece ser que el ejercicio de las opciones se realizó de una manera relativamente acelerada, en relación a lo que indica la función de ejercicio óptimo descrita en este trabajo.

iv) Por último, la ganancia promedio de haber observado la estrategia teórica, habría ascendido a $0.59 \%$, mientras que la prima teórica habría sido de $0.49 \%$. Este balance nos indica que nuestro proceso de valuación resultó bastante atinado durante este período. 


\section{V.- Conclusiones:}

En este trabajo se realizó una descripción del esquema de acumulación de reservas internacionales implementado por el Banco de México a través de la subasta de opciones de venta de dólares al propio banco central. Posteriormente se desarrolló un modelo de valuación de esta opción y se estableció la función de ejercicio óptimo. Con base en este modelo se desarrollaron las propiedades principales del mecanismo de intervención o de compra de divisas.

Los resultados de este ejercicio indican que el precio de la opción es creciente en la volatilidad del tipo de cambio y decreciente en la tendencia hacia la depreciación del tipo de cambio. En segundo lugar, se observó que cuando al inicio del período la restricción de que para que la opción pueda ejercerse el tipo de cambio debe ser menor (más apreciado) que su promedio móvil de los últimos 20 días, no opera, la probabilidad de ejercicio de la opción es alta y no depende de una manera importante ni de la volatilidad ni de la tendencia del tipo de cambio. Por lo tanto, este mecanismo de acumulación de reservas internacionales es neutral con respecto a dichos parámetros.

Finalmente se comparó la valuación y el ejercicio teórico de la opción con las observaciones de mercado. En dicha comparación se hace evidente una subvaluación del precio de la opción y una ejercicio anticipado de la opción por parte de dueños de las opciones. Esta subvaluación puede explicarse parcialmente si se supone que los individuos anticipan que se presente una depreciación brusca del tipo de cambio con una probabilidad pequeña. 


\section{Apéndice 1}

La siguiente ecuación se estimó por Mínimos Cuadrados Ordinarios.

$$
\left(M_{t}-S_{t}\right)=\alpha\left(M_{t-1}-S_{t-1}\right)+\beta \cdot \Delta S
$$

Lo anterior, con el fin de determinar los coeficientes $\alpha$ y $\beta$ para el periodo 1996-1997. Los resultados se muestran a continuación.

\begin{tabular}{crrr} 
Coeficiente & Valor & Error Est. & Est. $\boldsymbol{t}$ \\
\hline $\boldsymbol{\alpha}$ & 0.929 & 0.002 & 496.2 \\
$\boldsymbol{\beta}$ & -0.953 & 0.004 & -244.1 \\
$\mathbf{R}^{2}$ & 0.999 & & \\
\hline
\end{tabular}

Asimismo, se realizó una prueba de estabilidad para los coeficientes.
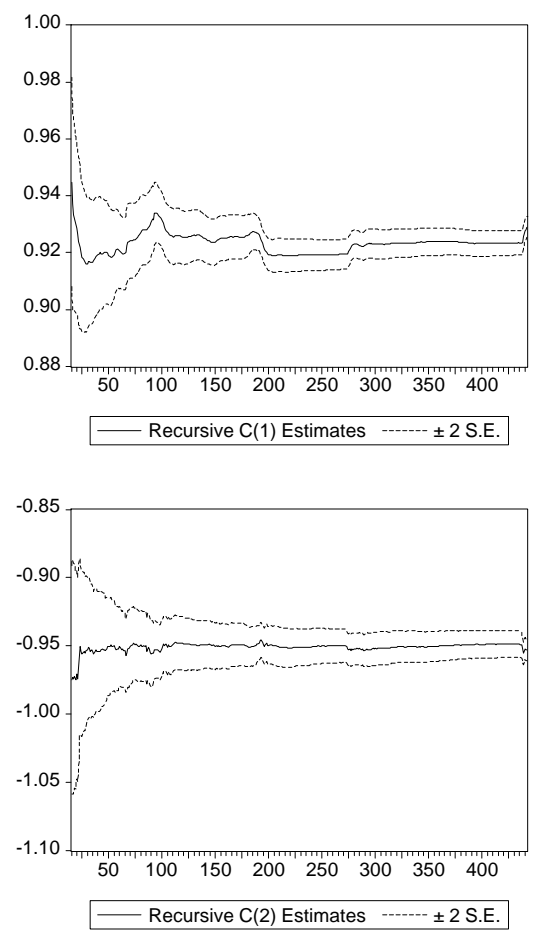

En la gráfica superior se muestra la prueba para el coeficiente $\alpha$ y en la inferior para el coeficiente $\beta$. Como se puede apreciar, ambos exhiben un comportamiento estable y la desviación estándar de cada uno decrece conforme se amplía la muestra. 


\section{VI.- Referencias:}

Galán, Manuel; Duclaud, J y García, A (1996) “ Una Estrategia de Acumulación de Reservas Mediante la Venta de Dólares: el caso de Banco de México" Mimeo, Banco de México.

Hull, John. (1993) "Options, Futures and other Derivative Securities" Prentice Hall.

Meese, Richard y Rogoff, K. (1983) "Empirical Exchange Rate Models of the Seventies, Do They Fit Out of Sample?". Journal of International Economics 14, Pgs. 3-24. Press.

Sargent, T. (1989) "Dynamic Macroeconomic Theory" Harvard University

Werner, Alejandro (1997) "Un Estudio Estadístico Sobre el Comportamiento de la Cotización del Peso Mexicano Frente al Dólar y de su Volatilidad". Gaceta de Economía ITAM, Año 3, Núm. 5, Suplemento. 